Interfaces and Free Boundaries 14 (2012), 307-342

DOI $10.4171 / \mathrm{IFB} / 283$

\title{
A two-phase problem with a lower-dimensional free boundary
}

\author{
MARK ALLEN \\ Department of Mathematics, Purdue University, West Lafayette, IN 47907, USA \\ E-mail: allenma@math.purdue.edu \\ ARShaK PETROSyAN \\ Department of Mathematics, Purdue University, West Lafayette, IN 47907, USA \\ E-mail:arshak@math.purdue.edu
}

[Received 20 May 2011 and in revised form 30 June 2012]

For a bounded domain $D \subset \mathbb{R}^{n}$, we study minimizers of the energy functional

$$
\int_{D}|\nabla u|^{2} d x+\int_{D \cap\left(\mathbb{R}^{n-1} \times\{0\}\right)} \lambda^{+} \chi_{\{u>0\}}+\lambda^{-} \chi_{\{u<0\}} d \mathcal{H}^{n-1},
$$

without any sign restriction on the function $u$. One of the main result states that the free boundaries

$$
\Gamma^{+}=\partial\{u(\cdot, 0)>0\} \quad \text { and } \quad \Gamma^{-}=\partial\{u(\cdot, 0)<0\}
$$

never touch. Moreover, using Alexandrov-type reflection technique, we can show that in dimension $n=3$ the free boundaries are $C^{1}$ regular on a dense subset.

\section{Mathematics Subject Classification: Primary 35R35.}

Keywords: Two-phase free boundary problem, lower-dimensional free boundary, separation of phases, regularity of the free boundary, monotonicity formula, Alexandrov reflection technique, Steiner symmetrization

\section{Introduction}

For a bounded domain $D$ in $\mathbb{R}^{n}$, consider the problem of minimizing the energy functional

$$
J(u)=\int_{D}|\nabla u|^{2} d x+\int_{D \cap\left(\mathbb{R}^{n-1} \times\{0\}\right)} \lambda^{+} \chi_{\{u>0\}}+\lambda^{-} \chi_{\{u<0\}} d \mathcal{H}^{n-1},
$$

among all functions $u \in W^{1,2}(D)$ with $u-u_{0} \in W_{0}^{1,2}(D)$ for a prescribed $u_{0}$. We assume that $\lambda^{+}$ and $\lambda^{-}$are positive constants. The main objective of this paper is to study the local properties of the free boundaries

$$
\Gamma^{ \pm}=\Gamma_{u}^{ \pm}:=\partial \Omega_{u}^{ \pm} \cap D, \quad \text { where } \Omega_{u}^{ \pm}:=\{ \pm u>0\} \cap\left(\mathbb{R}^{n-1} \times\{0\}\right) .
$$

The boundary here is defined by the topology of $\mathbb{R}^{n-1} \times\{0\}$, so formally it is of co-dimension two in $\mathbb{R}^{n}$.

This problem bears resemblance to the one of minimizing the functional

$$
\tilde{J}(u)=\int_{D}|\nabla u|^{2}+\lambda^{+} \chi_{\{u>0\}}+\lambda^{-} \chi_{\{u<0\}},
$$


studied in [2], the same paper where the renown Alt-Caffarelli-Friedman monotonicity formula has been introduced. The minimizers of (1.2) are generalized solutions of a classical two-phase free boundary problem

$$
\begin{aligned}
\Delta u=0 & \text { in }\{u>0\} \cup\{u<0\}, \\
\left|\nabla u_{+}\right|^{2}-\left|\nabla u_{-}\right|^{2}=M & \text { on } \partial\{u>0\} \cup \partial\{u<0\},
\end{aligned}
$$

with $M=\lambda^{+}-\lambda^{-}$. In one particular application, the problem (1.3) appears in a simplified model for premixed equidiffusional flames, in the stationary case. More specifically, one considers the limits as $\epsilon \rightarrow 0+$ of a singular perturbation problem

$$
\Delta u=\beta_{\epsilon}(u) \text { in } D
$$

where the nonlinearities $\beta_{\epsilon}, \epsilon>0$, are supported in $[0, \epsilon]$ and have a fixed total energy $\int_{0}^{\epsilon} \beta_{\epsilon}(s) d s=$ $M / 2$, see, e.g., [5].

When long range interactions are present, it is relevant to replace the Laplacian by nonlocal operators, such as the fractional Laplacian. See survey papers [13] and [3]. If one formally considers the equation

$$
\left(-\Delta_{x^{\prime}}\right)^{\alpha} u=-\beta_{\epsilon}(u) \text { on } \mathbb{R}^{n-1},
$$

where $\Delta_{x^{\prime}}$ is the Laplacian on $\mathbb{R}^{n-1}$ and $0<\alpha<1$, then in the case $\alpha=1 / 2$ this equation can be rewritten as a boundary reaction problem

$$
\begin{aligned}
& \Delta u=0 \text { in } \mathbb{R}^{n-1} \times(0, \infty), \\
& \partial_{x_{n}} u=\beta_{\epsilon}(u) \quad \text { on } \mathbb{R}^{n-1} \times\{0\},
\end{aligned}
$$

solutions of which can be found by minimizing a suitably smoothed version of the energy functional (1.1) on $\mathbb{R}^{n}$. Letting $\epsilon \rightarrow 0+$, we obtain thereby that the minimization problem (1.1) can be viewed as a "localized" version of the free boundary problem (1.3) for the half Laplacian $\left(-\Delta_{x^{\prime}}\right)^{1 / 2}$.

The one-phase version of our problem (i.e., nonnegative minimizers) has been recently considered in [8]. The authors of [8], in fact, consider the analogous problem for all fractional powers of Laplacian, by using the extension of Caffarelli and Silvestre [7]. While our study of the two-phase problem is only for $\alpha=1 / 2$, there are more technical tools available at our disposal (such as the Alt-Caffarelli-Friedman monotonicity formula) which allows us to obtain richer results.

\section{Main results and outline of paper}

The main results obtained in this paper are as follows.

- Existence of minimizers. In Section 2 we show the existence of minimizers (Theorem 2.1), including the maximal and minimal ones for the given boundary data (Theorem 2.3).

- Optimal regularity. In Section 3, we show that the bounded minimizers are in fact $C^{1 / 2}$ regular (Theorem 3.1). This is the best regularity possible since in fact $C \operatorname{Re}\left(x_{n-1}+i\left|x_{n}\right|\right)^{1 / 2}$ is a minimizer for appropriately chosen constant $C$ (Theorem 9.1).

- Convergence properties. Having the optimal regularity, in Section 4 we study the convergence properties of sequences of minimizers (Theorem 4.1), including the strong convergence in $W^{1,2}$ (Theorem 4.2). 
- Nondegeneracy. In Section 5 we show that the minimizers cannot decay faster than the square root of the distance from the free boundaries, in both phases, (Theorem 5.1), even restricted to the thin space $D^{\prime}=D \cap \mathbb{R}^{n-1} \times\{0\}$ (Theorem 5.5). As a consequence, we obtain that $\Omega_{u}^{ \pm}$satisfy a $\mathcal{H}^{n-1}$-density property (Theorem 5.7), which implies that $\mathcal{H}^{n-1}\left(\Gamma^{ \pm}\right)=0$.

- Separation of phases. In Section 6 we prove an unexpected result that the two phases $\Omega_{u}^{+}$and $\Omega_{u}^{-}$are separated in a sense that $\Gamma^{+} \cap \Gamma^{-}=\emptyset$, and that in fact the minimizers don't change sign in solid neighborhoods of points on $\Gamma^{ \pm}$(Theorem 6.1). This effectively reduces the twophase problem to an one-phase problem, at least for the study of the local properties of the free boundary. The proof is obtained by the application of Alt-Caffarelli-Friedman monotonicity formula.

This result is in complete contrast with two-phase free-boundary problem (1.3), where the twophase points create a major complication even in the proof of the optimal (Lipschitz in that case) regularity of solutions, see [2].

- $H^{n-3 / 2}$ measure of the free boundary. In Section 7 we show that the free boundary has $\mathcal{H}^{n-3 / 2}$ measure zero (Theorem 7.1). This result is not optimal, but it is a simple corollary for an estimate on $\mathcal{H}^{n-1}$-density of $\Delta u$ on $D^{\prime}$ (Lemma 7.2), that is instrumental for the remaining part of the paper.

- Monotonicity formula and blow-ups. In Section 8 we prove a Weiss-type monotonicity formula (Theorem 8.1). It has an immediate corollary that the blow-ups are homogeneous of degree $1 / 2$, see Section 9. We then give a characterization of so-called regular free boundary points (i.e., the points where the blow-up has a flat free boundary) in terms of the Weiss energy (Theorem 9.4). The proofs are heavily based on the use of the Steiner symmetrization.

- Regularity of the free boundary in dimension $n=3$. In Section 10 we prove that the set of regular points is a relatively open subset of the free boundary, and is locally a $C^{1}$ curve (Theorem 10.1). We do this only in dimension $n=3$ (so that the free boundary is contained in $\mathbb{R}^{2} \times\{0\}$ ), where we may apply the Alexandrov reflection technique, appropriately adapted to our setting.

\section{Notation and terminology}

Throughout the paper we will use the following notation.

- We denote a point $x \in \mathbb{R}^{n}$ by $\left(x^{\prime}, x_{n}\right)$ where $x^{\prime}=\left(x_{1}, \ldots, x_{n-1}\right)$.

- For $s \in \mathbb{R}$, we define $s_{ \pm}=\max ( \pm s, 0)$, the positive and negative parts of $s$, so that we have $s=s_{+}-s_{-}$.

- For any set $\Omega \subset \mathbb{R}^{n}$, we define

$$
\Omega^{\prime}:=\Omega \cap\left(\mathbb{R}^{n-1} \times\{0\}\right)
$$

We will refer to $\mathbb{R}^{n-1} \times\{0\}$ as the thin space.

- The balls $B_{r}(x)=\left\{y \in \mathbb{R}^{n}|| x-y \mid<r\right\}$ will be often referred to as solid balls; whereas, $B_{r}^{\prime}\left(x^{\prime}\right)=\left\{y^{\prime} \in \mathbb{R}^{n-1}|| y^{\prime}-x^{\prime} \mid<r\right\}$ will be referred to as the thin balls. Sometimes we will abuse the notation and write $B_{r}^{\prime}\left(x^{\prime}, 0\right)$ for $B^{\prime}\left(x^{\prime}\right) \times\{0\}$ and will identify $\mathbb{R}^{n-1}$ with $\mathbb{R}^{n-1} \times\{0\} \subset$ $\mathbb{R}^{n}$.

- The unit sphere in dimension $n$ will be denoted by $S^{n-1}$.

- The spherical coordinates $\left(r, \theta_{1}, \ldots, \theta_{n-1}\right) \in(0, \infty) \times[-\pi, \pi) \times[0, \pi] \times \cdots \times[0, \pi]$ for a nonzero 
point $x=\left(x_{1}, x_{2}, \ldots, x_{n}\right)$ are defined by the relations

$$
\begin{aligned}
r & =|x|, \\
x_{n} & =r \cos \theta_{n-1}, \\
& \cdots \\
x_{n-k} & =r \sin \theta_{n-1} \sin \theta_{n-2} \cdots \sin \theta_{n-k} \cos \theta_{n-k-1}, \\
& \cdots \\
x_{1} & =r \sin \theta_{n-1} \sin \theta_{n-2} \cdots \sin \theta_{1} .
\end{aligned}
$$

- We will call the sets $\Omega_{u}^{ \pm}:=\{ \pm u>0\} \cap\left(\mathbb{R}^{n-1} \times\{0\}\right)$ positive and negative phases of $u$ and $\Lambda_{u}:=\{u=0\} \cap\left(\mathbb{R}^{n-1} \times\{0\}\right)$ the zero set. The free boundary $\Gamma_{u}$ is the union of $\Gamma_{u}^{+}$and $\Gamma_{u}^{-}$, where $\Gamma_{u}^{ \pm}:=\partial \Omega_{u}^{ \pm} \cap D$.

- It is useful when studying local properties of free boundary points to consider the rescalings at $x_{0} \in \Gamma_{u}$

$$
u_{r}:=\frac{u\left(r x+x_{0}\right)}{r^{1 / 2}} .
$$

It is easy to see that the rescalings are still minimizers of the functional $J$. When we let $r \rightarrow 0$, the process is known as blow-up. If for a certain subsequence $r=r_{k} \rightarrow 0, u_{r}$ converges to $u_{0}$ (in a certain sense) we will also refer to $u_{0}$ as a blow-up of $u$ at $x_{0}$.

\section{Existence}

We say that $u$ is a minimizer of the functional $J$ in (1.1) if

$$
J(u) \leqslant J(v), \quad \text { for } v \in u+W_{0}^{1,2}(D) .
$$

Many of the results in this paper can be generalized also for local minimizers, for which (2.1) is satisfied with $v$ such that $\operatorname{supp}(u-v) \Subset D$ and diam $\operatorname{supp}(u-v)<\delta$ for some $\delta>0$.

Throughout the paper we will assume that the domain $D$ is bounded and that the subdomains $D^{ \pm}=D \cap\left\{ \pm x_{n}>0\right\}$ have Lipschitz boundaries. This guarantees, for instance, that the trace operator $W^{1,2}(D) \rightarrow L^{2}\left(D^{\prime}\right)$ is compact.

The next theorem establishes the existence of minimizers with a given Sobolev trace on $\partial D$.

THEOREM 2.1 (Existence) For any $\phi \in W^{1,2}(D)$ there exists a minimizer $u$ to the functional $J$ in the class $\mathfrak{K}=\phi+W_{0}^{1,2}(D)$.

Proof. Since $J(v) \geqslant 0$, there exists a minimizing sequence $\left\{u_{k}\right\}$ such that $J\left(u_{k}\right) \rightarrow \inf _{\mathfrak{K}} J$. Since $\left\|\nabla u_{k}\right\|_{L^{2}(D)}$ is bounded, and $\left.u_{k}\right|_{\partial D}=\phi$, we obtain that

$$
\left\|u_{k}\right\|_{W^{1,2}(D)} \text { is bounded. }
$$

Hence, we may extract a further subsequence such that

$$
u_{k} \rightarrow u \text { in } W^{1,2}(D) .
$$

It is clear that $u-\phi \in W_{0}^{1,2}(D)$, or, equivalently, $u \in \mathfrak{K}$. Furthermore, since the trace operator $\left.v \mapsto v\right|_{D^{\prime}}$ is compact, we may pass to a further subsequence to obtain

$$
u_{k} \rightarrow u \text { in } L^{2}\left(D^{\prime}\right), \quad \text { and } \quad u_{k} \rightarrow u \quad H^{n-1} \text {-a.e. in } D^{\prime} .
$$


Then there exist two functions $\gamma^{ \pm}$with $0 \leqslant \gamma^{ \pm} \leqslant 1$ such that

$$
\chi\left\{u_{k}>0\right\} \stackrel{*}{\rightarrow} \gamma^{+} \text {and } \quad \chi\left\{u_{k}<0\right\} \stackrel{*}{\rightarrow} \gamma^{-} .
$$

Since $\int_{D^{\prime}}\left(u_{k}\right)_{ \pm}\left(1-\chi_{\left\{ \pm u_{k}>0\right\}}\right)=0$, passing to the limit, we obtain $\int_{D^{\prime}} u_{ \pm}\left(1-\gamma^{ \pm}\right)=0$, which implies that

$$
\chi_{\{u>0\}} \leqslant \gamma^{+}, \quad \chi_{\{u<0\}} \leqslant \gamma^{-} \quad \mathcal{H}^{n-1} \text {-a.e. in } D^{\prime} .
$$

We then obtain:

$$
\begin{aligned}
\int_{D}|\nabla u|^{2} & +\int_{D^{\prime}} \lambda^{+} \chi\{u>0\}+\lambda^{-} \chi_{\{u<0\}} \\
& \leqslant \lim _{k \rightarrow \infty} \int_{D}\left|\nabla u_{k}\right|^{2}+\int_{D^{\prime}} \lambda^{+} \gamma^{+}+\lambda^{-} \gamma^{-} \\
& =\lim _{k \rightarrow \infty} \int_{D}\left|\nabla u_{k}\right|^{2}+\lim _{k \rightarrow \infty} \int_{D^{\prime}} \lambda^{+} \chi_{\left\{u_{k}>0\right\}}+\lambda^{-} \chi_{\left\{u_{k}<0\right\}} .
\end{aligned}
$$

Hence $u$ is a minimizer.

Note that since the functional $J$ is not convex, we may not necessarily have the uniqueness of the solution, and in general we may not necessarily conclude that if $u$ and $v$ are two minimizers with $u \leqslant v$ on $\partial D$, then $u \leqslant v$ in $D$. Instead we have the following lemma.

LEMMA 2.2 (Lattice property) Let $u, v$ be two minimizers of the functional $J$ in a domain $D$ with $\left.u\right|_{\partial D} \leqslant\left. v\right|_{\partial D}$. If we define $\bar{w} \equiv \max \{u, v\}$ and $\underline{w} \equiv \min \{u, v\}$, then $\bar{w}$ and $\underline{w}$ are minimizers of the functional $J$ with boundary values $v$ and $u$ respectively.

Proof. It is fairly straightforward to check that

$$
J(\bar{w})+J(\underline{w})=J(u)+J(v) .
$$

Since $\left.\bar{w}\right|_{\partial D}=v$ and $\left.\underline{w}\right|_{\partial D}=u$, we conclude that $\bar{w}$ and $\underline{w}$ are minimizers of the functional $J$.

THEOREM 2.3 (Maximal and minimal minimizers) For any $\phi \in W^{1,2}(D)$ there exists a maximal (minimal) minimizer $u^{*}\left(u_{*}\right)$ of $J$ on $D$ with boundary data $\phi$ on $\partial D$ such that $v \leqslant u^{*}\left(v \geqslant u_{*}\right)$ for all other minimizers $v$ with $\left.v\right|_{\partial D}=\phi$.

Moreover, if $u_{1}^{*}$ and $u_{2}^{*}$ are maximal minimizers corresponding to boundary data $\phi_{1}$ and $\phi_{2}$ on $\partial D$ and such that $\phi_{1} \leqslant \phi_{2}$ then $u_{1}^{*} \leqslant u_{2}^{*}$. A similar statement holds for minimal minimizers.

Proof. The existence of $u^{*}$ and $u_{*}$ is obtained by the limiting procedure by using the lattice property, similar to the standard Perron method.

The second part of the theorem is a direct consequence of the lattice property.

COROLlary 2.4 If $D$ and the boundary data $\phi$ on $\partial D$ are axially symmetric about the line $\left(0, \ldots, 0, x_{n}\right)$, then so will be $u^{*}$ and $u_{*}$.

Proof. By Theorem 2.3, if $O$ is any rotation about the line $\left(0, \ldots, 0, x_{n}\right)$, then $u^{*} \circ O$ is a minimizer with the same boundary data and therefore $u^{*} \circ O \leqslant u^{*}$. This is possible only if $u^{*}$ is axially symmetric about $\left(0, \ldots, 0, x_{n}\right)$. The same proof holds for $u_{*}$. 


\section{Optimal regularity}

In this section we show the Hölder- $1 / 2$ regularity of minimizers. This regularity is suggested by the natural scaling of the problem. Namely, if $u$ is a minimizer of $J$, then $u_{r}(x)=u\left(r x+x_{0}\right) / r^{1 / 2}$ is still a minimizer of $J$ in the appropriately scaled domain.

THEOREM 3.1 (Hölder-1/2 regularity) Let $u$ be a minimizer of $J$ in $B_{1}$ with $\|u\|_{L^{\infty}\left(\partial B_{1}\right)} \leqslant M$. Then

$$
\|u\|_{C^{1 / 2}\left(B_{1 / 2}\right)} \leqslant C
$$

where $C$ is a constant depending only on $n, M$, and $\lambda^{ \pm}$.

Remark 3.2. In the above theorem we only need to control $\|u\|_{L^{\infty}}$ on the boundary of the ball, since it is straightforward to show that if $\|u\|_{L^{\infty}\left(\partial B_{1}\right)} \leqslant M$, then $\|u\|_{L^{\infty}\left(B_{1}\right)} \leqslant M$. Similarly, we note that if $\left.u\right|_{\partial B_{1}} \geqslant 0(\leqslant 0)$ then $u \geqslant 0(\leqslant 0)$ in all of $B_{1}$.

To prove the above theorem, we will need a Caccioppoli inequality. Without assuming a priori that $u$ is continuous, we do not necessarily know that $u_{+}$and $u_{-}$are subharmonic. Instead we prove the Caccioppoli inequality directly from the fact that $u$ is a minimizer.

LEMMA 3.3 Let $u$ be a minimizer of the functional $J$ in $B_{2 r}$. Then

$$
\int_{B_{r}}|\nabla u|^{2} \leqslant \frac{C_{n}}{r^{2}} \int_{B_{2 r}} u^{2}
$$

where $C_{n}$ is a constant depending only on the dimension $n$.

Proof. Choose a cut-off function $\eta \in C_{0}^{\infty}\left(B_{2 r}\right)$ such that

$$
0 \leqslant \eta \leqslant 1, \quad \eta \equiv 1 \text { on } B_{r}, \quad|\nabla \eta| \leqslant \frac{C_{n}}{r}
$$

and consider a competing function $u^{\epsilon}=u-\epsilon u_{+} \eta^{2}$ for a small $\epsilon>0$. Note that $\left\{u^{\epsilon}>0\right\}=\{u>0\}$ and $\left\{u^{\epsilon}<0\right\}=\{u<0\}$, besides $u^{\epsilon}=u$ on $\partial B_{2 r}$. Therefore, from the minimality of $u$, we must have

$$
\int_{B_{2 r}}|\nabla u|^{2} \leqslant \int_{B_{2 r}}\left|\nabla\left(u-\epsilon u_{+} \eta^{2}\right)\right|^{2},
$$

which by letting $\epsilon \rightarrow 0+$ yields

$$
\int_{B_{2 r}} \nabla u \nabla\left(u+\eta^{2}\right) \leqslant 0 .
$$

Proceeding as in the standard proof of the Caccioppoli inequality, we arrive at

$$
\int_{B_{r}}\left|\nabla u_{+}\right|^{2} \leqslant \frac{C_{n}}{r^{2}} \int_{B_{2 r}} u_{+}^{2} .
$$

Similar inequality holds also for $u_{-}$. This completes the proof of the lemma.

We are now able to prove the optimal regularity of minimizers. 
Proof of Theorem 3.1. Let $B_{r}(x)$ be a ball contained in $B_{1}$. Consider the harmonic replacement $v$ of $u$ in $B_{r}(x)$; i.e., a harmonic function $v$ in $B_{r}(x)$ such that $v=u$ on $\partial B_{r}(x)$. Then, using that

$$
\int_{B_{r}(x)} \nabla v \nabla(v-u)=0
$$

combined with the minimality of $u$ in $B_{1}$, we obtain the estimate

$$
\begin{aligned}
\int_{B_{r}(x)} & |\nabla(u-v)|^{2}=\int_{B_{r}(x)}|\nabla u|^{2}-|\nabla v|^{2} \\
\quad & \int_{B_{r}(x) \cap\left(\mathbb{R}^{n-1} \times\{0\}\right)} \lambda^{+}\left(\chi_{\{v>0\}}-\chi_{\{u>0\}}\right)+\lambda^{-}\left(\chi_{\{v<0\}}-\chi_{\{u<0\}}\right) \\
\leqslant & C_{0} r^{n-1},
\end{aligned}
$$

with $C_{0}$ depending only on $n$ and $\lambda^{ \pm}$. Then for any $0<\rho<r$ we have

$$
\begin{aligned}
\left(\int_{B_{\rho}(x)}|\nabla u|^{2}\right)^{1 / 2} & \leqslant\left(\int_{B_{r}(x)}|\nabla(u-v)|^{2}\right)^{1 / 2}+\left(\int_{B_{\rho}(x)}|\nabla v|^{2}\right)^{1 / 2} \\
& \leqslant C_{0} r^{(n-1) / 2}+\left(\frac{\rho}{r}\right)^{n / 2}\left(\int_{B_{r}(x)}|\nabla v|^{2}\right)^{1 / 2} \\
& \leqslant C_{0} r^{(n-1) / 2}+\left(\frac{\rho}{r}\right)^{n / 2}\left(\int_{B_{r}(x)}|\nabla u|^{2}\right)^{1 / 2}
\end{aligned}
$$

Here in the second inequality we have used that $t^{-n} \int_{B_{t}(x)}|\nabla v|^{2}$ is nondecreasing for $0<t<r$, because of the subharmonicity of $|\nabla v|^{2}$ in $B_{r}(x)$, and in the third inequality we have used that $v$ minimizes the Dirichlet integral on $B_{r}(x)$. The above estimate can be rewritten as

$$
A(\rho) \leqslant C_{0}\left(\frac{r}{\rho}\right)^{(n-1) / 2}+\left(\frac{\rho}{r}\right)^{1 / 2} A(r),
$$

where

$$
A(r):=\left(\frac{1}{r^{n-1}} \int_{B_{r}(x)}|\nabla u|^{2}\right)^{1 / 2} .
$$

Choosing $\rho=\delta r$ for a fixed $0<\delta<1 / 2$, and using a simple induction argument that starts with the Caccioppoli inequality for $B_{\delta}(x)$, we arrive at the estimate

$$
A\left(\delta^{k}\right) \leqslant C, \quad k=1,2, \ldots,
$$

where $C=C\left(C_{0}, \delta, M\right)$ is a large constant satisfying

$$
C \geqslant C_{0} \delta^{-(n-1) / 2} /(1-\delta), \quad C \geqslant C_{n} M \delta^{-1 / 2} .
$$

This readily implies that

$$
\int_{B_{r}(x)}|\nabla u|^{2} \leqslant C r^{n-1}
$$

for any $x \in B_{1 / 2}$ and $r \leqslant 1 / 2$. Applying Morrey's theorem, see e.g., [11, Theorem 1.53], we conclude that $u$ is Hölder- $1 / 2$. 
Remark 3.4. Now that we know that minimizers are continuous and we may use first variation to conclude that $u$ is harmonic on the set $\{u \neq 0\} \cup D^{+} \cup D^{-}$. In particular, we also obtain that $u_{+}$ and $u_{-}$are continuous subharmonic functions in entire $D$.

\section{Convergence of minimizers}

In this section we have collected some results on the convergence of sequences of minimizers, that are going to be important in blow-up analysis and compactness type arguments throughout the paper.

THEOREM 4.1 (Convergence of minimizers) Let $\left\{u_{k}\right\}$ be a sequence of minimizers of the functional $J$ in the domain $D$ with $\left\|u_{k}\right\|_{L^{\infty}(\partial D)} \leqslant M$. Then there exists a subsequence and a function $u_{0}$ such that for every open $U \Subset D$

$$
\begin{aligned}
& \text { (1) } u_{0} \in W^{1,2}(U) \cap C^{1 / 2}(\bar{U}), \\
& \text { (2) } u_{k} \rightarrow u_{0} \text { in } C^{\alpha}(\bar{U}) \text { for } \alpha<1 / 2 \\
& \text { (3) } u_{k} \rightarrow u_{0} \text { in } W^{1,2}(U) \\
& \text { (4) } u_{0} \text { is a minimizer of } J \text { in } U
\end{aligned}
$$

Proof. Properties (1)-(3) follow immediately from Lemma 3.3 and Theorem 3.1. So we will concentrate on the proof of (4). We must show $J\left(u_{0}\right) \leqslant J\left(u_{0}+\psi\right)$ for $\psi \in W_{0}^{1,2}(U)$. Since minimizers exist and are Hölder-1/2 continuous, we only have to show the inequality for $\psi$ continuous. Choose a cut-off function $\eta \in C_{0}^{\infty}(D)$ such that

$$
0 \leqslant \eta \leqslant 1, \quad \eta \equiv 1 \text { on a neighborhood of } \bar{U} .
$$

For the minimizer $u_{k}$ consider the following competing function:

$$
v_{k}^{\epsilon}=\left(u_{k}+\psi-\epsilon \eta\right)_{+}-\left(u_{k}+\psi+\epsilon \eta\right)_{-} .
$$

Then we have

$$
\begin{aligned}
J\left(u_{k}\right) \leqslant & J\left(v_{k}^{\epsilon}\right) \\
\leqslant & \int_{D}\left|\nabla\left(u_{k}+\psi\right)\right|^{2}+\int_{\operatorname{supp} \eta \backslash \bar{U}}\left(2 \epsilon\left|\nabla u_{k}\right||\nabla \eta|+\epsilon^{2}|\nabla \eta|^{2}\right) \\
& \quad+\int_{D^{\prime}} \lambda^{+} \chi_{\left\{u_{k}+\psi-\epsilon \eta>0\right\}}+\lambda^{-} \chi_{\left\{u_{k}+\psi+\epsilon \eta<0\right\}} .
\end{aligned}
$$

We now want to pass to the limits as $k \rightarrow \infty$. To this end, subtract

$$
\int_{D}\left|\nabla u_{k}\right|^{2}+\int_{D^{\prime} \backslash \bar{U}} \lambda^{+} \chi_{\left\{u_{k}>0\right\}}+\lambda^{-} \chi_{\left\{u_{k}<0\right\}}
$$

from both sides of the previous inequality to obtain

$$
\begin{aligned}
\int_{D^{\prime} \cap \bar{U}} \lambda^{+} \chi_{\left\{u_{k}>0\right\}}+\lambda^{-} \chi_{\left\{u_{k}<0\right\}} & \leqslant \int_{D}\left(2 \nabla u_{k} \nabla \psi+|\nabla \psi|^{2}\right) \\
& +\int_{D^{\prime} \cap \bar{U}} \lambda^{+} \chi_{\left\{u_{k}+\psi-\epsilon>0\right\}}+\lambda^{-} \chi_{\left\{u_{k}+\psi+\epsilon<0\right\}}+C \epsilon .
\end{aligned}
$$


Now, using that $\nabla u_{k} \rightarrow \nabla u_{0}$ in $L^{2}(D)$ and $u_{k} \rightarrow u_{0}$ uniformly on $\bar{U}$, we obtain

$$
J\left(u_{0}\right) \leqslant \int_{D}\left|\nabla\left(u_{0}+\psi\right)\right|^{2}+\int_{D^{\prime}} \lambda^{+} \chi_{\left\{u_{0}+\psi>0\right\}}+\lambda^{-} \chi_{\left\{u_{0}+\psi<0\right\}}+C \epsilon .
$$

Letting $\epsilon \rightarrow 0$, we conclude that $J\left(u_{0}\right) \leqslant J\left(u_{0}+\psi\right)$.

Our next result strengthens the convergence given in part (4) of Theorem 4.1 from weak convergence in $W^{1,2}$ to strong convergence, so minimizers under the assumptions of Theorem 4.1 will be locally compact in $W^{1,2}$.

THEOREM 4.2 (Strong convergence) Let $\left\{u_{k}\right\}$ and $u_{0}$ be as in Theorem 4.1. Then, over a subsequence, $u_{k} \rightarrow u_{0}$ strongly in $W^{1,2}(U)$ for any open $U \Subset D$.

To prove this theorem, we will need the following result on the structure of $\Delta u$ for the minimizer $u$.

LEMma 4.3 Let $u$ be a minimizer of the functional $J$. Then $\Delta u$ is a signed Radon measure supported in $\Lambda=\{u=0\} \cap D^{\prime}$ with the total variation $|\Delta u|$ satisfying

$$
\left\langle|\Delta u|, \chi_{K}\right\rangle \leqslant C(n, K)\|\nabla u\|_{L^{2}(D)}
$$

for any $K \Subset D$. Moreover, $\Delta u$ is absolutely continuous with respect to $\left.\mathcal{H}^{n-1}\right|_{\Lambda}$ and

$$
\Delta u=\left.\omega \mathcal{H}^{n-1}\right|_{\Lambda \backslash \Gamma}, \quad \omega=\frac{\partial u}{\partial x_{n}}+\frac{\partial u}{\partial x_{-n}}
$$

or equivalently, for $\phi \in C_{0}^{\infty}(D)$,

$$
\langle\Delta u, \phi\rangle=\int_{\Lambda \backslash \Gamma} \phi \omega d \mathcal{H}^{n-1} .
$$

In the statement of the lemma above we have used the notation

$$
\begin{gathered}
\frac{\partial u}{\partial x_{n}}\left(x_{0}, 0\right)=\lim _{h \rightarrow 0} \frac{u\left(x_{0}, h\right)-u\left(x_{0}, 0\right)}{h}, \\
\frac{\partial u}{\partial x_{-n}}\left(x_{0}, 0\right)=\lim _{h \rightarrow 0} \frac{u\left(x_{0},-h\right)-u\left(x_{0}, 0\right)}{-h},
\end{gathered}
$$

where $h>0$, which exist at every point $x_{0} \in D^{\prime} \backslash \Gamma$.

Proof. The functions $u_{ \pm}$are nonnegative, continuous, harmonic where positive. Hence, $u_{ \pm}$are subharmonic in $B_{1}$, implying that $\Delta u_{ \pm}$are nonnegative Radon measures, and consequently that $\Delta u=\Delta u_{+}-\Delta u_{-}$is a signed Radon measure. Besides, we know that $u$ is harmonic in $B_{1} \backslash \Lambda$ which implies that $\Delta u$ lives on $\Lambda$. The quantitative estimate on $|\Delta u|$ follows from a standard argument for subharmonic functions. Indeed, let $\eta \in C_{0}^{\infty}(D)$ be a nonnegative cut-off function such that

$$
\eta \equiv 1 \quad \text { on } K, \quad\|\nabla \eta\|_{L^{2}(D)} \leqslant C(n, K) .
$$

Then,

$$
\left\langle\Delta u_{ \pm}, \chi_{K}\right\rangle \leqslant\left\langle\Delta u_{ \pm}, \eta\right\rangle=-\int_{D} \nabla u_{+} \nabla \eta \leqslant C(n, K)\|u\|_{L^{2}(D)}
$$


and the claimed estimate follows.

For the second part of the theorem, we will essentially prove the divergence theorem directly. If fact, we will need to jump ahead and use the fact that $\mathcal{H}^{n-1}(\Gamma)=0$, see Corollary 5.8. (We just note here that the proof of Theorem 5.7 and Corollary 5.8 is independent of Lemma 4.3.)

We first break up the integral over $D$ as follows:

$$
\int_{D} u \Delta \phi=\int_{D_{+}} u \Delta \phi+\int_{D_{-}} u \Delta \phi
$$

Then, we break up the Laplacian as:

$$
\int_{D_{+}} u \Delta \phi=\int_{D_{+}} u \frac{\partial^{2} \phi}{\partial x_{n}^{2}}+\sum_{i=1}^{n-1} \int_{D_{+}} u \frac{\partial^{2} \phi}{\partial x_{i}^{2}} .
$$

Further, use iterated integrals as follows:

$$
\int_{D_{+}} u \frac{\partial^{2} \phi}{\partial x_{n}^{2}}=\int_{D^{\prime} \backslash \Gamma} \int_{0}^{\infty} u \frac{\partial^{2} \phi}{\partial x_{n}^{2}} .
$$

(We may integrate over $D^{\prime} \backslash \Gamma$ since $\mathcal{H}^{n-1}(\Gamma)=0$ ). We are now able to use integration by parts on each line to obtain

$$
\int_{D^{\prime} \backslash \Gamma} \int_{0}^{\infty} u \frac{\partial^{2} \phi}{\partial x_{n}^{2}}=\int_{D^{\prime} \backslash \Gamma}\left(\frac{\partial u}{\partial x_{n}} \phi-\frac{\partial \phi}{\partial x_{n}} u\right)+\int_{D_{+}} \frac{\partial^{2} u}{\partial x_{n}^{2}} \phi .
$$

Since also

$$
\sum_{i=1}^{n-1} \int_{D_{+}} u \frac{\partial^{2} \phi}{\partial x_{i}^{2}}=\sum_{i=1}^{n-1} \int_{D_{+}} \phi \frac{\partial^{2} u}{\partial x_{i}^{2}}
$$

combining the equalities above, we arrive at

$$
\int_{D_{+}} u \Delta \phi=\int_{D_{+}} \phi \Delta u+\int_{D^{\prime} \backslash \Gamma}\left(\frac{\partial u}{\partial x_{n}} \phi-\frac{\partial \phi}{\partial x_{n}} u\right) .
$$

Now, $u$ is harmonic and differentiable off the zero set, so when we add the integral over $D_{-}$, we obtain

$$
\int_{D} u \Delta \phi=\int_{\Lambda \backslash \Gamma}\left(\frac{\partial u}{\partial x_{n}}+\frac{\partial u}{\partial x_{-n}}\right) \phi .
$$

We can now prove the strong convergence.

Proof of Theorem 4.2. Take a test function $\eta \in C_{0}^{\infty}(D)$ such that

$$
0 \leqslant \eta \leqslant 1, \quad \eta \equiv 1 \text { in a neighborhood of } \bar{U} .
$$

Next, suppose that $k$ is so large that $\left|u_{k}-u\right|<\epsilon$ on supp $\eta$. Then we will have

$$
\left|\left\langle\Delta\left(u_{k}-u\right),\left(u_{k}-u\right) \eta^{2}\right\rangle\right| \leqslant \epsilon\left(\left\langle\left|\Delta u_{k}\right|, \eta^{2}\right\rangle+\left\langle|\Delta u|, \eta^{2}\right\rangle\right) \leqslant C \epsilon,
$$


where $C$ depends only on $L^{2}$ norms of $\nabla u_{k}$ and $\nabla u$ on $D$, by Lemma 4.3. Therefore

$$
\int_{D}\left|\nabla\left(u_{k}-u\right)\right|^{2} \eta^{2} \leqslant C \epsilon-2 \int_{D} \eta\left(u_{k}-u\right)\left\langle\nabla\left(u_{k}-u\right), \nabla \eta\right\rangle
$$

and applying Young's inequality, we arrive at

$$
\int_{U}\left|\nabla\left(u_{k}-u\right)\right|^{2} \leqslant C \epsilon+C \int_{\operatorname{supp} \eta}\left(u_{k}-u\right)^{2} .
$$

One of the main applications of the results of this section is to the existence of blow-ups at free boundary points.

COROLLARY 4.4 (Existence of blow-ups) Let $u$ be a minimizer $x_{0} \in \Gamma_{u}$. Then the family of rescalings $\left\{u_{r}\right\}$ defined by

$$
u_{r}(x)=\frac{u\left(x_{0}+r x\right)}{r^{1 / 2}}, \quad x \in \frac{1}{r}\left(D-x_{0}\right)
$$

is uniformly bounded on every $U \Subset \mathbb{R}^{n}$ for $0<r<r_{U}$. In particular, there exists a subsequence $r_{j} \rightarrow 0$ and a function $u_{0}$ on $\mathbb{R}^{n}$ such that $u_{r_{j}} \rightarrow u_{0}$ on every $U \Subset \mathbb{R}^{n}$, in the senses described in Theorems 4.1 and 4.2. The function $u_{0}$ is a called a blow-up of $u$ at $x_{0}$ and is a minimizer on every $U \Subset \mathbb{R}^{n}$.

Proof. Just observe that if $\delta>0$ is such that $B_{\delta}\left(x_{0}\right) \subset D$, then $u_{r}$ is defined at least in $B_{\delta / r}$ and, by Theorem 3.1, the family $\left\{u_{r}\right\}$ is uniformly bounded on $B_{R}$ for $0<r<\delta / R$. The rest follows from Theorems 4.1 and 4.2.

\section{Nondegeneracy}

When we consider blow-ups of minimizers at free boundary points, it is not immediately obvious that they may not vanish identically in $\mathbb{R}^{n}$. What is even less obvious is whether the origin will be a free boundary point for the blow-ups. The main results of this section, Theorems 5.1 and 5.5, address these issues.

THEOREM 5.1 (Nondegeneracy) Fix $0<t<1$, and let $u$ be a minimizer of $J$ in $B_{r}$. There exists $\epsilon>0$ with $\epsilon$ depending only on $\left\{\lambda^{+}, \lambda^{-}, t\right\}$ such that if $\left.u\right|_{\partial B_{r}} \leqslant \epsilon r^{1 / 2}\left(\left.u\right|_{\partial B_{r}} \geqslant-\epsilon r^{1 / 2}\right)$ then

$$
u(x) \leqslant 0, \quad(u(x) \geqslant 0) \quad \text { for } x \in B_{t r}^{\prime} .
$$

To prove this theorem, we will need the following estimate.

Lemma 5.2 Fix $0<\kappa<1$. If $u$ is a minimizer on $B_{1}$ with $u \leqslant M$ on $\partial B_{1}$, then

$$
\int_{B_{\kappa}^{\prime}} \lambda^{+} \chi_{\{u>0\}} \leqslant M^{2} C_{n, \kappa},
$$

where $C_{n, \kappa}$ is a constant depending only on dimension $n$ and $\kappa$.

Proof. For $0<\kappa<1$, let $\phi_{\kappa}$ be the solution to

$$
\left.\phi_{\kappa}\right|_{\partial B}=1,\left.\quad \phi_{\kappa}\right|_{B_{\kappa}^{\prime}}=0, \quad \Delta \phi_{\kappa}(x)=0 \text { for } x \notin B_{\kappa}^{\prime} .
$$


We first note that the solution $\phi_{\kappa}$ exists since $B \backslash B_{\kappa}^{\prime}$ is a regular domain for the Dirichlet problem by the Wiener criterion. Let $v \equiv M \phi_{\kappa}$ and $w \equiv \min \{u, v\}$. Then $J(u) \leqslant J(w)$, and by grouping similar terms we find that

$$
\int_{D^{\prime} \cap\{v=0\}} \lambda^{+} \chi\{u>0\} \leqslant \int_{D \cap\{u>v\}}|\nabla v|^{2}-|\nabla u|^{2}
$$

Note that we have used that $u^{-}=w^{-}$. Now we use that $u$ is harmonic in the open set $\{u>v\}$ so that

$$
\int_{\{u>v\}}|\nabla u|^{2}=\int_{\{u>v\}} \nabla u \nabla v
$$

Substituting this into inequality (5.1) gives

$$
\int_{D^{\prime} \cap\{v=0\}} \lambda^{+} \chi_{\{u>0\}} \leqslant \int_{\{u>v\}} \nabla v \nabla(v-u)=\int_{B_{\kappa}^{\prime}}(\Delta v) u=\langle\Delta v, u\rangle .
$$

Here, $\Delta v$ is a nonnegative Radon measure in $B_{1}$ whose support is $\{v=0\}=B_{\kappa}^{\prime}$ (see, e.g., proof of Lemma 4.3). The lemma now follows from the bound

$$
\langle\Delta v, u\rangle \leqslant M^{2}\left\langle\Delta \phi_{\kappa}, \chi_{B_{\kappa}^{\prime}}\right\rangle \leqslant M^{2} C_{n, \kappa}
$$

Next lemma improves the statement of Corollary 2.4 when the boundary data is constant.

LEMMA 5.3 Let $u$ be a minimizer of $J$ on $B_{1}$ such that the values of $\left.u\right|_{\partial B_{1}}=M>0$. Then $u$ is symmetric about the line $\left(0, \ldots, 0, x_{n}\right)$, and the coincidence set $\Lambda=\{u=0\} \cap B_{1}^{\prime}$ is connected and centered at the origin.

Proof. First observe that $0 \leqslant u \leqslant M$ in $B_{1}$, see Remark 3.2. Extend $u$ to be a function on the cube $Q_{1}$ with side length 2 , by defining $u(x)=M$ for $x \notin B_{1}$. Now, fix a direction $e \in \mathbb{R}^{n-1} \times\{0\}$ and apply Steiner symmetrization (as defined in [10, Section II.7]) to the function $v=M-u$ on lines parallel to $e$ and let $\tilde{v}$ denote the resulting function. If we only consider $\left\{x \in Q_{1}|| x_{n} \mid>\epsilon\right\}$, then $v$ is Lipschitz. Then by [10, Theorem 2.31], we have that

$$
\int_{B_{1} \cap\left\{\left|x_{n}\right|>\epsilon\right\}}|\nabla u|^{2}=\int_{B_{1} \cap\left\{\left|x_{n}\right|>\epsilon\right\}}|\nabla v|^{2} \geqslant \int_{B_{1} \cap\left\{\left|x_{n}\right|>\epsilon\right\}}|\nabla \tilde{v}|^{2}
$$

and by letting $\epsilon \rightarrow 0$

$$
\int_{B_{1}}|\nabla u|^{2}=\int_{B_{1}}|\nabla v|^{2} \geqslant \int_{B_{1}}|\nabla \tilde{v}|^{2} .
$$

If now $\tilde{u}=M-\tilde{v}$, then by the properties of Steiner symmetrization, we also have

$$
\mathcal{H}^{n-1}\left(\{u>0\} \cap B_{1}^{\prime}\right)=\mathcal{H}^{n-1}\left(\{\tilde{u}>0\} \cap B_{1}^{\prime}\right) .
$$

Therefore we obtain that $J(\tilde{u}) \leqslant J(u)$ and since $u=\tilde{u}$ on $\partial B_{1}$, we conclude that $\tilde{u}$ is also a minimizer. Now, we want to show that in fact $\tilde{u} \equiv u$. To this end, consider $\bar{w}=\max \{u, \tilde{u}\}$, which is also a minimizer by Lemma 2.2. In particular, $\bar{w}$ is harmonic in $B_{1} \backslash\{\bar{w}=0\} \supset B_{1} \backslash B_{1}^{\prime}$. Then by the strict maximum principle for harmonic functions, we have that either $u \equiv \bar{w}$ or $\tilde{u} \equiv \bar{w}$ in 
$B_{1}^{ \pm}$, and hence in all of $B_{1}$. This is equivalent to having $u \leqslant \tilde{u}$ or $\tilde{u} \leqslant u$ in $B_{1}$. In the first case, the equality

$$
\int_{B_{1} \backslash\{\tilde{u}=0\}}|\nabla \tilde{u}|^{2}=\int_{B_{1} \backslash\{\tilde{u}=0\}}|\nabla u|^{2}
$$

and the harmonicity of $\tilde{u}$ in $B_{1} \backslash\{\tilde{u}=0\}$ implies that $u$ is also harmonic there and consequently that $u \equiv \tilde{u}$ in this case. The second case is treated similarly, implying that indeed $\tilde{u} \equiv u$. To complete the proof, we Steiner symmetrize along all directions $e \in \mathbb{R}^{n-1} \times\{0\}$ to obtain that $u$ is symmetric about the line $\left(0, \ldots, 0, x_{n}\right)$ and $\{u=0\}$ is connected and centered at the origin.

We are now able to prove the nondegeneracy result.

Proof of Theorem 5.1. First we note that by rescaling we only need to prove Theorem 5.1 on the unit ball $B_{1}$. Also, Theorem 2.3 reduces Theorem 5.1 to proving the theorem for the maximal minimizer $u_{\epsilon}^{*}$ where $\left.u_{\epsilon}^{*}\right|_{\partial B}=\epsilon$. Lemma 5.3 proves that $\left\{u_{\epsilon}^{*}=0\right\}=B_{\rho}^{\prime}$ for some $\rho<1$. Lemma 5.2 shows

$$
\int_{B_{\kappa}^{\prime}} \lambda^{+} \chi_{\left\{u_{\epsilon}^{*}>0\right\}} \rightarrow 0 \quad \text { as } \quad \epsilon \rightarrow 0 \text {. }
$$

Then there exists $\epsilon$ depending only on $\left\{t, \lambda^{+}\right\}$such that if $\left.u\right|_{\partial B_{1}}=\epsilon$ then

$$
\left.u\right|_{B_{t}^{\prime}}=0 .
$$

The case for which $u \geqslant-\epsilon$ is proven similarly.

COROLlaRy 5.4 If $u$ is a minimizer and $0 \in \Gamma^{+}\left(0 \in \Gamma^{-}\right)$, then

$$
\sup _{\partial B_{r}} u \geqslant C r^{1 / 2} \quad\left(\inf _{\partial B_{r}} u \leqslant-C r^{1 / 2}\right),
$$

where $C$ depends only on $\lambda^{+}, \lambda^{-}$and $n$.

We have nondegeneracy for the solid balls. The next theorem will give us nondegeneracy in the thin space. This result will have many implications in the study of the free boundary. We note that Theorems 5.5 and 5.7 have already been proven in [8] for the one-phase problem.

THEOREM 5.5 (Nondegeneracy in the thin space) Let $u$ be a minimizer of $J$ in $B_{r}$ with $\|u\|_{C^{1 / 2}\left(B_{r}\right)} \leqslant M$. Then there exists $C>0$ and $0<\rho<1$, depending only on $n, M$, and $\lambda^{+}$ $\left(\lambda^{-}\right)$, such that if $0 \in \overline{\Omega_{u}^{+}}\left(0 \in \overline{\Omega_{u}^{-}}\right)$then

$$
\sup _{B_{r}^{\prime} \backslash B_{\rho r}^{\prime}} u \geqslant C r^{1 / 2} \quad\left(\inf _{B_{r}^{\prime} \backslash B_{\rho r}^{\prime} r} u \leqslant-C r^{1 / 2}\right) .
$$

Proof. By rescaling, it is enough to prove the lemma for the case when $r=1$. Suppose first that $0 \in \overline{\Omega_{u}^{+}}$and $u \leqslant 0$ on $B_{1}^{\prime} \backslash B_{\rho}^{\prime}$ with $\rho<1 / 4$ to be chosen later. Let $\phi_{1 / 2}$ be as defined in Lemma 5.2. Then, as before:

$$
\lambda^{+} \int_{B_{1 / 2}^{\prime}} \chi_{\{u>0\}} \leqslant M \int_{B_{1 / 2}^{\prime}}\left(\Delta \phi_{1 / 2}\right) u
$$


Now, $\Delta \phi_{1 / 2}$ is a nonnegative Radon measure, with a support in $\bar{B}_{1 / 2}^{\prime}$ and in fact

$$
\Delta \phi_{1 / 2}=\left.2 \frac{\partial \phi_{1 / 2}}{\partial x_{n}} \mathcal{H}^{n-1}\right|_{B_{1 / 2}^{\prime}}
$$

Moreover, it is easy to see that $\partial \phi_{1 / 2} / \partial x_{n} \leqslant C_{n}$ on $B_{1 / 4}^{\prime} \supset B_{\rho}^{\prime}$. Using also that $u \leqslant M \rho^{1 / 2}$ on $B_{\rho}^{\prime}$, we can write

$$
\lambda^{+} \int_{B_{1 / 2}^{\prime}} \chi_{\{u>0\}} \leqslant C_{n} M \rho^{1 / 2} \int_{B_{1 / 2}^{\prime}} \chi_{\{u>0\}} .
$$

Thus, by making $\rho$ small enough, we would obtain that $u \leqslant 0$ on $B_{\rho}^{\prime}$. This would be a contradiction since $0 \in \Gamma^{+}$.

Suppose, by way of contradiction, that there exists a sequence $\left\{u_{k}\right\}$ of minimizers in $B_{1}$ with $\left\|u_{k}\right\|_{C^{1 / 2}\left(B_{1}\right)} \leqslant M, 0 \in \overline{\Omega_{u_{k}}^{+}}$, and such that

$$
u_{k}\left(x^{\prime}, 0\right)<\frac{1}{k}\left|x^{\prime}\right|^{1 / 2}, \quad \text { for all }\left(x^{\prime}, 0\right) \text { with } \rho \leqslant\left|x^{\prime}\right| \leqslant 1 .
$$

Then $u_{k} \rightarrow u_{0}$ in $C^{\alpha}$ for $\alpha<1 / 2$. Furthermore, by Theorem 4.1, $u_{0}$ is a minimizer of $J$ in any ball $B_{r}, 0<r<1$. Since $0 \in \overline{\Omega_{u_{k}}^{+}}$, then $u_{0}$ inherits the same nondegeneracy properties of Theorem 5.1 that each $u_{k}$ has. Then $0 \in \overline{\Omega_{u_{0}}^{+}}$. But in the limit $u_{0} \leqslant 0$ on $B_{1}^{\prime} \backslash B_{\rho}^{\prime}$. This is a contradiction.

The nondegeneracy in the thin space has one immediate corollary. We omit the simple proof.

COROLlaRY 5.6 Let $\left\{u_{k}\right\}$ and $u_{0}$ be as in Theorem 4.1. If $x_{k} \in \Gamma_{u_{k}}^{ \pm}$and $x_{k} \rightarrow x_{0} \in D$, then $x_{0} \in \Gamma_{u_{0}}^{ \pm}$.

We next show the positive density of the free boundary.

TheOREM 5.7 Let $u$ be a minimizer of $J$ in $B_{1}$ with $\|u\|_{C^{1 / 2}\left(B_{1}\right)} \leqslant M$ and $0 \in \Gamma^{+}$. Then there exists $c=c\left(n, M, \lambda^{+}\right)>0$ such that

$$
c<\frac{\mathcal{H}^{n-1}\left(\Omega_{u}^{+} \cap B_{r}^{\prime}\right)}{\mathcal{H}^{n-1}\left(B_{r}^{\prime}\right)}<1-c
$$

for every $0<r<1$. Similar estimate holds also for $\Omega_{u}^{-}$if $0 \in \Gamma^{-}$.

Proof. Since $0 \in \Gamma^{+}$, by Theorem 5.5 there exists $\left(x^{\prime}, 0\right)$ such that $\left|x^{\prime}\right|>\rho r$ and $u\left(x^{\prime}, 0\right) \geqslant$ $C\left|x^{\prime}\right|^{1 / 2}$. By uniform Hölder-regularity, $u$ will be positive in a small ball around $\left(x^{\prime}, 0\right)$. This proves the estimate from below in (5.4).

To prove the estimate from above, we note that if $B_{r / 2}^{\prime} \cap \Omega_{u}^{-}$is nonempty, then arguing as in the preceding paragraph, we obtain that the set $\Omega_{u}^{-} \cap B_{r}^{\prime} \subset B_{r}^{\prime} \backslash \Omega_{u}^{+}$is large enough, which proves the estimate in this case. So it remains to consider the case when $u \geqslant 0$ in $B_{r / 2}^{\prime}$. Besides, scaling if necessary, we may assume that $r=1 / 2$. Now, if the estimate from above fails in this case, we can find a sequence of minimizers $u_{k}$ as in the statement of the theorem such that $u_{k} \geqslant 0$ in $B_{1 / 4}^{\prime}$ and

$$
\mathcal{H}^{n-1}\left(\left\{u_{k}=0\right\} \cap B_{1 / 4}^{\prime}\right) \rightarrow 0
$$


Let now $v_{k}$ be a harmonic function in $B_{1 / 4}$, with the same boundary values as $u_{k}$ on $\partial B_{1 / 4}$. Then arguing as in the beginning of the proof of Theorem 3.1, we will have

$$
\int_{B_{1 / 4}}\left|\nabla\left(v_{k}-u_{k}\right)\right|^{2} \leqslant \lambda^{+} \int_{B_{1 / 4}^{\prime}} \chi_{\left\{u_{k}=0\right\}} \rightarrow 0 .
$$

Next, passing to a subsequence, we may assume that $u_{k} \rightarrow u_{0}$ and $v_{k} \rightarrow v_{0}$ uniformly in $B_{1 / 4}$. Clearly, $v_{0}$ is harmonic in $B_{1 / 4}$ and $u_{0}$ is a minimizer of $J$ in $B_{\rho}$ for $\rho<1 / 4$, with $0 \in \Gamma_{u_{0}}^{+}$. Besides, by Fatou's lemma $\nabla u_{0}=\nabla v_{0}$ in $B_{1 / 4}$, which implies that $u_{0} \equiv v_{0}+c$. Consequently, $u_{0}$ is also harmonic in $B_{1 / 4}$. Now observe that $u_{0} \geqslant 0$ in $B_{1 / 4}$ and $u_{0}(0)=0$. By the strong maximum principle this implies that $u_{0} \equiv 0$ in $B_{1 / 4}$. However, this contradicts the fact that $0 \in \Gamma_{u_{0}}^{+}$.

COROLlary 5.8 The free boundaries $\Gamma^{ \pm}$have $\mathfrak{H}^{n-1}$ measure zero.

Proof. Apply Lebesgue's density theorem and use the property (5.4).

The zero $\mathfrak{H}^{n-1}$ measure of the free boundaries allows to conclude the following fact about the convergence of the positivity and negativity sets of minimizers.

THEOREM 5.9 Let $\left\{u_{k}\right\}$ and $u_{0}$ be as in Theorem 4.1. Then, over a subsequence,

$$
\chi\left\{u_{k}>0\right\} \rightarrow \chi\left\{u_{0}>0\right\}, \quad \chi\left\{u_{k}<0\right\} \rightarrow \chi\left\{u_{0}<0\right\} \quad H^{n-1} \text {-a.e. on } D^{\prime} .
$$

Proof. Without loss of generality we may assume that $D$ is the unit ball $B_{1}$ and prove only that $\chi_{\left\{u_{k}>0\right\}} \rightarrow \chi_{\left\{u_{0}>0\right\}} \mathcal{H}^{n-1}$-a.e. on $B_{1 / 2}^{\prime}$. We will also assume that the properties (1)-(4) in Theorem 4.1 hold.

First, if $x \in\left\{u_{0}>0\right\} \cap B_{1 / 2}^{\prime}$, then from $C^{\alpha}$ convergence, $u_{k}(x)>0$ for $k$ sufficiently large.

Next, if $x \in B_{1 / 2}^{\prime}$ and $u_{0} \leqslant 0$ on $B_{\delta}^{\prime}(x)$, then we claim that $u_{k} \leqslant 0$ on $B_{\delta / 2}^{\prime}(x)$ for $k$ sufficiently

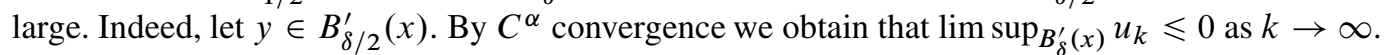
By Theorem 5.5 there exists $C$ such that if for all $z \in B_{\delta / 4}^{\prime}(y)$

$$
u_{k}(z)<C(\rho \delta / 4)^{1 / 2}
$$

then $y$ cannot be in $\overline{\Omega_{u_{k}}^{+}}$. Thus, $u_{k} \leqslant 0$ on $B_{\delta / 2}^{\prime}(x)$ if $k$ is large enough, as claimed.

Hence, we have established that $\chi_{\left\{u_{k}>0\right\}} \rightarrow \chi_{\left\{u_{0}>0\right\}}$ everywhere on $B_{1 / 2}^{\prime} \backslash \Gamma_{u_{0}}^{+}$. Since $\mathcal{H}^{n-1}\left(\Gamma_{u_{0}}^{+}\right)=0$, this completes the proof of the theorem.

\section{Separation of phases}

In this section we prove that the free boundaries $\Gamma^{+}, \Gamma^{-}$cannot meet and that effectively near the free boundary we deal only with a one phase problem.

THEOREM 6.1 (Separation of phases) Let $u$ be a minimizer of $J$. Then $\Gamma^{+} \cap \Gamma^{-}=\emptyset$, i.e., the free boundaries $\Gamma^{+}$and $\Gamma^{-}$cannot touch. Moreover, for any $x_{0} \in \Gamma^{+}\left(x_{0} \in \Gamma^{-}\right)$there exists an open ball $B_{t}\left(x_{0}\right) \subset D$ such that $u \geqslant 0(u \leqslant 0)$ in $B_{t}\left(x_{0}\right)$.

As we will see, this follows from the combination of the nondegeneracy in Theorem 5.1 and the following monotonicity formula. 
LEMMA 6.2 (Alt-Caffarelli-Friedman monotonicity formula) Let $\left\{h_{1}, h_{2}\right\}$ be a pair of nonnegative continuous subharmonic functions in $B_{1}$ such that $h_{1} \cdot h_{2}=0$ in $B_{1}$. Then the functional

$$
r \mapsto \Phi\left(r, h_{1}, h_{2}\right)=\frac{1}{r^{4}} \int_{B_{r}} \frac{\left|\nabla h_{1}\right|^{2}}{|x|^{n-2}} \int_{B_{r}} \frac{\left|\nabla h_{2}\right|^{2}}{|x|^{n-2}}
$$

is finite and nondecreasing for $0<r<1$.

This monotonicity formula has first appeared in [2]. See also [6, Chapter 12] for more details. (There is a superfluous assumption $h_{1}(0)=h_{2}(0)$ in some versions of this monotonicity formula which can be readily discarded). In a typical application we will have $h_{1}=u_{+}$and $h_{2}=u_{-}$for a minimizer $u$, which satisfy the assumptions of the monotonicity formula, see Remark 3.4. Using the scaling properties of the functional $\Phi$, we can then obtain the following statement about the blow-ups.

LEMMA 6.3 Let $u_{0}$ be a blow-up of a minimizer $u$ at $x_{0} \in \Gamma_{u}$ over a sequence $r=r_{k} \rightarrow 0$, as described in Corollary 4.4. Then

$$
\int_{B_{R}} \frac{\left|\nabla\left(u_{0}\right)+\right|^{2}}{|x|^{n-2}} \int_{B_{R}} \frac{\left|\nabla\left(u_{0}\right)_{-}\right|^{2}}{|x|^{n-2}}=0,
$$

for any $R>0$. Thus, either $\left(u_{0}\right)_{+} \equiv 0$ or $\left(u_{0}\right)_{-} \equiv 0$ in all of $\mathbb{R}^{n}$.

Proof. Without loss of generality assume $x_{0}=0$ and let $u_{r}(x)=u(r x) / r^{1 / 2} \rightarrow u_{0}(x)$ over the sequence $r=r_{k} \rightarrow 0$. Making a simple change of variables and using the monotonicity of $\Phi$, we obtain

$$
\Phi\left(R,\left(u_{r}\right)_{+},\left(u_{r}\right)_{-}\right)=r^{2} \Phi\left(r R, u_{+}, u_{-}\right) \leqslant r^{2} \Phi\left(1, u_{+}, u_{-}\right) \rightarrow 0 \quad \text { as } r \rightarrow 0 .
$$

Then, taking $r=r_{k}$ and applying Fatou's lemma, in the limit we obtain that $\Phi\left(R,\left(u_{0}\right)_{+},\left(u_{0}\right)_{-}\right)=$ 0 for any $R>0$. This may happen only if either $\left(u_{0}\right)_{+}$or $\left(u_{0}\right)_{-}$is identically constant in $\mathbb{R}^{n}$. Since $u_{0}(0)=0$, this constant must be zero, and therefore one of the functions $\left(u_{0}\right)_{ \pm}$must vanish identically in $\mathbb{R}^{n}$.

Proof of Theorem 6.1. We split the proof into two steps.

(1) Thin separation: $\Gamma^{+} \cap \Gamma^{-}=\emptyset$.

Suppose by way of contradiction, that $0 \in \Gamma^{+} \cap \Gamma^{-}$. Consider then a blow-up $u_{r_{k}} \rightarrow u_{0}$. By Corollary 5.4, there exists $C>0$ such that for every $k$ there are points $x_{k}, y_{k} \in \partial B_{1}$ such that $u_{r_{k}}\left(x_{k}\right) \geqslant C$ and $u_{r_{k}}\left(y_{k}\right) \leqslant-C$. We may further assume that $x_{k} \rightarrow x_{0}$ and $y_{k} \rightarrow y_{0}$, passing to a subsequence, if necessary. Then, since the convergence $u_{r_{k}} \rightarrow u_{0}$ can be assumed locally uniform in $\mathbb{R}^{n}$, we immediately obtain $u_{0}\left(x_{0}\right) \geqslant C$ and $u_{0}\left(y_{0}\right) \leqslant-C$. However, this contradicts Lemma 6.3. Hence, the free boundaries $\Gamma^{+}$and $\Gamma^{-}$cannot meet.

(2) Solid separation: If $0 \in \Gamma^{+}$, then there exists $t>0$ such that $u \geqslant 0$ for all $x \in B_{t}$.

In the previous step we have essentially showed that $u$ has a sign in the thin ball $B_{t}^{\prime}$ for a small $t$. Here we show that $u$ has a sign in the solid ball $B_{t}$.

Let $u_{r_{k}} \rightarrow u_{0}$ be a blow-up of $u$ at the origin. By Lemma 6.3, $u_{0} \geqslant 0$. Since each $u_{r_{k}}\left(x^{\prime}, x_{n}\right)$ is harmonic in $B_{1 / r_{k}} \backslash B_{1 / r_{k}}^{\prime}$, then $u_{0}$ will be harmonic in the open set $\mathbb{R}_{ \pm}^{n}$. We define

$$
\delta=\inf u_{0} \text { over the set } B_{1} \cap\left\{\left|x_{n}\right| \geqslant 1 / 2\right\} .
$$


We claim that $\delta>0$. Indeed, otherwise by the strong minimum principle $u_{0} \equiv 0$ in $\mathbb{R}_{+}^{n}$ or $\mathbb{R}_{-}^{n}$, and therefore $u_{0} \equiv 0$ on $\mathbb{R}^{n-1} \times\{0\}$, which contradicts the fact that $0 \in \Gamma_{u_{0}}^{+}$(see Corollary 5.6). Then, by $C^{\alpha}$ convergence, for large enough $k, u_{r_{k}}\left(x^{\prime}, x_{n}\right) \geqslant \delta / 2$ for $\left|x_{n}\right| \geqslant 1 / 2$ in $B_{1}$. Also by $C^{\alpha}$ convergence, $\inf _{B_{1}} u_{r_{k}} \rightarrow 0$. Now by thin separation, for large enough $k$,

$$
u_{r_{k}}\left(x^{\prime}, 0\right) \geqslant 0 \text { in } B_{1}^{\prime} .
$$

Without loss of generality it suffices to show that $u_{r_{k}} \geqslant 0$ in $B_{1 / 2}^{+}$. Let $v_{k}$ be the harmonic function such that

$$
\left.v_{k}\right|_{B_{1}^{\prime}}=0 \text {, and }\left.v_{k}\right|_{\partial B_{1}^{+}}=u_{r_{k}} \text {. }
$$

Then $v_{k} \leqslant u_{r_{k}}$ in all of $B_{1}^{+}$. We show for $k$ large enough that $v_{k} \geqslant 0$ in $B_{1 / 2}^{+}$. To this end, consider two subsets $E_{1}$ and $E_{2}$ of $\partial\left(B_{1}^{+}\right)$:

$$
E_{1}=\partial\left(B_{1}^{+}\right) \cap\left\{x_{n} \geqslant 1 / 2\right\}, \quad E_{2}=\partial\left(B_{1}^{+}\right) \cap\left\{0<x_{n}<1 / 2\right\},
$$

and there harmonic measures $\omega_{1}$ and $\omega_{2}$ with respect to the domain $B_{1}^{+}$. The latter means that $\omega_{i}$ are harmonic functions in $B_{1}^{+}$satisfying

$$
\left.\omega_{i}\right|_{\partial\left(B_{1}^{+}\right)}=\chi_{E_{i}}, \quad i=1,2 .
$$

By using an explicit representation with the Poisson kernel or the boundary Harnack inequality, one then has that

$$
c_{n} x_{n} \leqslant \omega_{i}(x) \leqslant C_{n} x_{n} \quad \text { in } B_{1 / 2}^{+} .
$$

for some positive dimensional constants $c_{n}$ and $C_{n}$. Now, by using the maximum principle we then can write that in $B_{1 / 2}^{+}$

$$
\begin{aligned}
v_{k}(x) & \geqslant(\delta / 2) \omega_{1}(x)+\omega_{2}(x) \inf _{B_{1}^{+}} v_{k} \\
& \geqslant x_{n}\left[(\delta / 2) c_{n}-C_{n} \sup _{\left(\partial B_{1}\right)^{+}} u_{r_{k}}^{-}\right] .
\end{aligned}
$$

Since $u_{r_{k}}^{-} \rightarrow 0$ uniformly on compact subsets of $\mathbb{R}^{n}$, we obtain that $v_{k}(x) \geqslant 0$ in $B_{1 / 2}^{+}$for large $k$. This completes the proof.

\section{7. $\mathcal{H}^{n-3 / 2}$ measure of the free boundary}

For the remainder of the paper we will consider local properties of the free boundary. Using the results of the previous section, it will suffice to assume unless otherwise stated that we now consider minimizers of the functional

$$
J(v)=\int_{D}|\nabla v|^{2}+\int_{D^{\prime}} \chi_{\{v>0\}}
$$

where $v \in \mathfrak{K}=\left\{w \in W^{1,2}|w|_{\partial D}=\phi\right\}$ and $\phi \geqslant 0$. Since there is no negative phase, it will be natural to denote the free boundary $\Gamma_{v}^{+}$simply by $\Gamma_{v}$.

We start with an improvement on Corollary 5.8. 
THEOREM 7.1 Let $u$ be a minimizer of $J$. Then

$$
\mathcal{H}^{n-3 / 2}(\Gamma)=0 .
$$

This result is far from being optimal, indeed, one would expect the free boundary to have locally finite $\mathcal{H}^{n-2}$ measure. We state it partially because its proof uses and estimate on the measure $\Delta u$ that we will apply more than once throughout this paper.

Lemma 7.2 Let $u$ be a minimizer with $\|u\|_{C^{1 / 2}(D)} \leqslant M$. For any compact $K \Subset D$, there exist two positive constants $c, C$ depending only on $n, M$ and $K$ such that if $\left(x^{\prime}, 0\right) \in(\Lambda \backslash \Gamma) \cap K$, then

$$
\frac{c}{\sqrt{\operatorname{dist}\left(x^{\prime}, \Gamma\right)}}<\frac{\partial u}{\partial x_{n}}\left(x^{\prime}, 0\right)<\frac{C}{\sqrt{\operatorname{dist}\left(x^{\prime}, \Gamma\right)}} .
$$

Proof. Without loss of generality we may assume $D=B_{1}$ and $K=\overline{B_{1 / 4}}$. Suppose by way of contradiction that there exists a sequence of minimizers $\left\{u_{k}\right\}$ with $\left\|u_{k}\right\|_{C^{\alpha}\left(\overline{B_{1}}\right)} \leqslant M$ and points $x_{k} \in\left(\Lambda_{u_{k}} \backslash \Gamma_{u_{k}}\right) \cap \overline{B_{1 / 4}}$ such that

$$
\frac{\partial u_{k}}{\partial x_{n}}\left(x_{k}\right) \leqslant \frac{1}{k \sqrt{\operatorname{dist}\left(x_{k}, \Gamma_{k}\right)}}
$$

By rescaling with

$$
\tilde{u}_{k}(x)=\frac{u_{k}\left(2 d_{k} x+y_{k}\right)}{\sqrt{2 d_{k}}}
$$

where $y_{k} \in \Gamma_{u_{k}}$ and $d_{k}=\operatorname{dist}\left(x_{k}, \Gamma\right)=\left|x_{k}-y_{k}\right|$, we obtain that $\left\{\tilde{u}_{k}\right\}$ is a uniformly bounded family of minimizers in $B_{1}$ with $0 \in \Gamma_{\tilde{u}_{k}}$. Then by Theorem 4.1, we may extract a subsequence $\tilde{u}_{k} \rightarrow u$, where $u$ is a minimizer on every ball $B_{r}, r<1$. Note that by Corollary 5.6, $u$ is not identically zero. Also, if we denote $\xi_{k}=\left(x_{k}-y_{k}\right) / 2 d_{k}$ then $\left|\xi_{k}\right|=1 / 2$ and we may also assume that $\xi_{k} \rightarrow \xi_{0}$ with $\left|\xi_{0}\right|=1 / 2$. Furthermore, since $\tilde{u}_{k}$ is harmonic in $B_{1 / 4}^{+}\left(\xi_{k}\right)$ and $\tilde{u}_{k}(x)=0$ for $x \in B_{1 / 4}^{\prime}\left(\xi_{k}\right)$, we obtain that $u$ is harmonic in $B_{1 / 4}^{+}\left(\xi_{0}\right)$ and $u(x)=0$ for $x \in B_{1 / 4}^{\prime}\left(\xi_{0}\right)$. By $C^{1}$ convergence up to the boundary for harmonic functions we obtain that

$$
\frac{\partial u}{\partial x_{n}}\left(\xi_{0}\right)=0
$$

This is a violation of the Hopf principle, and we arrive at a contradiction. The proof of the estimate from above is similar. Only this time we suppose that

$$
\frac{\partial u_{k}}{\partial x_{n}}\left(x_{k}\right)>k \frac{1}{\sqrt{\operatorname{dist}\left(x_{k}, \Gamma\right)}} .
$$

This time, in the limit we obtain that

$$
\frac{\partial u}{\partial x_{n}}\left(\xi_{0}\right)=\infty,
$$

which is of course a contradiction. This completes the proof.

We are now able to prove Theorem 7.1. 
Proof of Theorem 7.1. Without loss of generality we will assume that $u$ is a minimizer on $B_{1}$ and show that $\mathcal{H}^{n-3 / 2}\left(\Gamma \cap B_{1 / 2}\right)=0$. By Lemmas 4.3 and 7.2, we know that as a measure for $x^{\prime} \in \Lambda$

$$
\frac{c}{\sqrt{\operatorname{dist}(x, \Gamma)}}<\frac{\partial u}{\partial x_{n}}\left(x^{\prime}, 0\right)+\frac{\partial u}{\partial x_{-n}}\left(x^{\prime}, 0\right)<\frac{C}{\sqrt{\operatorname{dist}(x, \Gamma)}} .
$$

Let $A_{\epsilon}=\left\{x^{\prime} \in \Lambda \mid 0<\operatorname{dist}\left(x^{\prime}, \Gamma\right)<\epsilon\right\}$. Then

$$
\mathcal{H}^{n-1}\left(A_{\epsilon}\right) \leqslant c \epsilon^{1 / 2}\left\langle\Delta u, A_{\epsilon}\right\rangle .
$$

Let $B=B_{1 / 2}$, and take a cover of $\Gamma \cap B$ by a collection thin balls $B_{i}^{\prime}$ of radius $\epsilon$ centered on the free boundary with the property that at most $N$ balls intersect. $N$ is dependent only on dimension. From Theorem 5.7 we have

$$
\mathcal{H}^{n-1}\left(B_{i}^{\prime} \cap A_{\epsilon}\right) \geqslant \beta \mathcal{H}^{n-1}\left(B_{i}^{\prime}\right)
$$

for some $\beta>0$ and therefore

$$
\begin{aligned}
\sum_{i} \mathcal{H}^{n-1}\left(B_{i}^{\prime}\right) & \leqslant \frac{1}{\beta} \sum_{i} \mathcal{H}^{n-1}\left(B_{i}^{\prime} \cap A_{\epsilon}\right) \\
& \leqslant \frac{N}{\beta} \mathcal{H}^{n-1}\left(B \cap A_{\epsilon}\right) \\
& \leqslant \frac{\left\langle\Delta u, B \cap A_{\epsilon}\right\rangle N c \epsilon^{1 / 2}}{\beta} \\
& \leqslant \frac{\langle\Delta u, B\rangle N c \epsilon^{1 / 2}}{\beta} .
\end{aligned}
$$

This shows that the $\mathcal{H}^{n-3 / 2}$-measure of $\Gamma \cap B$ is finite. To show that actually $\mathcal{H}^{n-3 / 2}(\Gamma \cap B)=0$, we just notice that

$$
\left\langle\Delta u, B \cap A_{\epsilon}\right\rangle \rightarrow 0 \quad \text { as } \epsilon \rightarrow 0
$$

due to the fact that $\Delta u$ is a Radon measure and $B \cap A_{\epsilon} \searrow \emptyset$.

\section{Weiss-type monotonicity formula}

In this section we establish a Weiss-type monotonicity formula, which will be a useful tool in the study of local properties of the free boundary. One of its immediate corollaries is the $1 / 2$ homogeneity of blow-ups. We prove this monotonicity formula for minimizers without a sign restriction.

THEOREM 8.1 (Weiss-type monotonicity formula) Let $u$ be a minimizer of $J$ as in (1.1) in $B_{R}\left(x_{0}\right)$ for $x_{0} \in \mathbb{R}^{n-1} \times\{0\}$. Define the Weiss energy functional

$$
\begin{aligned}
W\left(r, u, x_{0}\right):= & \frac{1}{r^{n-1}}\left(\int_{B_{r}\left(x_{0}\right)}|\nabla u|^{2}+\int_{B_{r}^{\prime}\left(x_{0}\right)} \lambda^{+} \chi_{\{u>0\}}+\lambda^{-} \chi_{\{u<0\}}\right) \\
& -\frac{1 / 2}{r^{n}} \int_{\partial B_{r}\left(x_{0}\right)} u^{2},
\end{aligned}
$$


for $0<r<R$. Then $W\left(r, u, x_{0}\right)$ is monotone nondecreasing in $r$. Furthermore, if $r_{1}<r_{2}$, then $W\left(r_{1}, u, x_{0}\right)=W\left(r_{2}, u, x_{0}\right)$ if and only if $u$ is homogeneous of degree $1 / 2$ with respect to $x_{0}$ on the ring $r_{1}<\left|x-x_{0}\right|<r_{2}$.

The latter means $u\left(x_{0}+\lambda y\right)=\lambda^{1 / 2} u\left(x_{0}+y\right)$, as long as $\lambda>0$ and $|y|, \lambda|y| \in\left(r_{1}, r_{2}\right)$.

Remark 8.2. Although, we may choose $x_{0}$ to be any point, it will be most useful to choose $x_{0} \in \Gamma$. We will also use a short-cut notation $W(r, u)$ when $x_{0}=0$.

Remark 8.3. The Weiss energy functional is essentially a normalized and boundary adjusted version of $J$. The normalization is chosen so that $W$ is preserved under the scaling $u_{r}(x)=u(r x) / r^{1 / 2}$ in the sense that $W(r, u)=W\left(1, u_{r}\right)$.

Proof. The proof is along the lines of that given by Weiss in [12]. Without loss of generality we may assume $x_{0}=0$. Let $\tau_{\epsilon}(x)=x+\epsilon \eta_{k} x$, where

$$
\eta_{k}(x)=\max \left\{0, \min \left\{1, \frac{r-|x|}{k}\right\}\right\} .
$$

Then $\eta_{k}(x)=0$ outside of $B_{r}(0)$, and

$$
\eta_{k}(x) \rightarrow \chi_{B_{r}(0)} \quad \text { as } k \rightarrow 0
$$

Notice that $\tau_{\epsilon}(x)=x\left(1+\epsilon \eta_{k}(x)\right)$ leaves $\mathbb{R}^{n-1} \times\{0\}$ invariant. We will also denote by $\tau_{\epsilon}^{\prime}$ the restriction of $\tau_{\epsilon}$ to $\mathbb{R}^{n-1} \times\{0\}$. Define now the function $u_{\epsilon}$ by the identity $u_{\epsilon}(y)=u(x)$, where $y=\tau_{\epsilon}(x)$. Then

$$
\frac{1}{\epsilon}\left(J\left(u_{\epsilon}\right)-J(u)\right) \geqslant 0
$$

and

$$
\begin{aligned}
& J\left(u_{\epsilon}\right)-J(u)=\int_{D}\left|\nabla u_{\epsilon}(y)\right|^{2}+\int_{D^{\prime}} \lambda^{+} \chi_{\left\{u_{\epsilon}>0\right\}}+\lambda^{-} \chi_{\left\{u_{\epsilon}<0\right\}} \\
&-\int_{D}|\nabla u(x)|^{2}-\int_{D^{\prime}} \lambda^{+} \chi_{\{u>0\}}+\lambda^{-} \chi_{\{u<0\}} .
\end{aligned}
$$

We explicitly have

$$
\begin{aligned}
& \nabla \eta_{k}(x)=\frac{-x}{|x| k} \chi_{B_{r} \backslash B_{r-k}}, \\
& D \tau_{\epsilon}(x)=I+\epsilon\left(\eta_{k}(x) I+x \nabla \eta_{k}(x)\right)
\end{aligned}
$$

and therefore

$$
\begin{aligned}
\operatorname{det} D \tau_{\epsilon}(x) & =1+\epsilon \operatorname{trace} D\left(\eta_{k}(x) x\right)+o(\epsilon), \\
D \tau_{\epsilon}^{-1} & =I-\epsilon D\left(\eta_{k}(x) x\right)+o(\epsilon) .
\end{aligned}
$$


Then, substituting these into the equality above, we obtain:

$$
\begin{aligned}
J\left(u_{\epsilon}\right)-J(u)= & \int_{D}\left|\nabla u(x)\left(D \tau_{\epsilon}(x)\right)^{-1}\right|^{2} \operatorname{det} D \tau_{\epsilon} \\
& +\int_{D^{\prime}}\left[\lambda^{+} \chi_{\{u>0\}}+\lambda^{-} \chi_{\{u<0\}}\right] \operatorname{det} D \tau_{\epsilon}^{\prime}(x) \\
& -\int_{D}|\nabla u|^{2}-\int_{D^{\prime}} \lambda^{+} \chi_{\{u>0\}}+\lambda^{-} \chi_{\{u<0\}} \\
= & \int_{D}\left[|\nabla u|^{2}-2 \epsilon \nabla u D\left(\eta_{k}(x) x\right) \nabla u\right]\left[1+\epsilon \operatorname{div}\left(\eta_{k}(x) x\right)\right]+o(\epsilon) \\
& +\int_{D^{\prime}}\left[\lambda^{+} \chi_{\{u>0\}}+\lambda^{-} \chi_{\{u<0\}}\right]\left[1+\epsilon \operatorname{div}\left(\eta_{k}^{\prime}\left(x^{\prime}, 0\right) x^{\prime}\right)\right]+o(\epsilon) \\
& -\int_{D}|\nabla u|^{2}-\int_{D^{\prime}} \lambda^{+} \chi_{\{u>0\}}+\lambda^{-} \chi_{\{u<0\}} \\
= & \int_{D} \epsilon|\nabla u|^{2} \operatorname{div} \eta_{k}(x) x-2 \epsilon \nabla u D\left(\eta_{k}(x) x\right) \nabla u+o(\epsilon) \\
& +\int_{D^{\prime}}\left[\lambda^{+} \chi_{\{u>0\}}+\lambda^{-} \chi_{\{u<0\}}\right]\left[\epsilon \operatorname{div}\left(\eta_{k}^{\prime}\left(x^{\prime}, 0\right) x^{\prime}\right)\right]+o(\epsilon) .
\end{aligned}
$$

Now, we may let $\epsilon$ be both positive and negative, implying that

$$
\lim _{\epsilon \rightarrow 0} \frac{1}{\epsilon}\left[J\left(u_{\epsilon}\right)-J(u)\right]=0 .
$$

Then we obtain the following equality:

$$
\begin{aligned}
0=\int_{D}|\nabla u|^{2} \operatorname{div} \eta_{k}(x) x-2 \nabla u D\left(\eta_{k}(x) x\right) & \nabla u \\
& +\int_{D^{\prime}}\left[\lambda^{+} \chi_{\{u>0\}}+\lambda^{-} \chi_{\{u<0\}}\right] \operatorname{div}\left(\eta_{k}^{\prime}\left(x^{\prime}, 0\right) x^{\prime}\right) .
\end{aligned}
$$

Using the explicit formulas

$$
\begin{aligned}
\operatorname{div}\left(\eta_{k}(x) x\right) & =n \eta_{k}(x)-\frac{|x|}{k} \chi_{B_{r} \backslash B_{r-k},}, \\
\operatorname{div}\left(\eta_{k}\left(x^{\prime}, 0\right) x^{\prime}\right) & =(n-1) \eta_{k}^{\prime}-\frac{\left|x^{\prime}\right|}{k} \chi_{B_{r}^{\prime} \backslash B_{r-k}^{\prime}},
\end{aligned}
$$

we obtain

$$
\begin{aligned}
0=(n-2) \int_{B_{r}}|\nabla u|^{2} \eta_{k} & -\frac{1}{k} \int_{B_{r} \backslash B_{r-k}}|x|\left[|\nabla u|^{2}-2\left(\frac{x}{|x|} \nabla u\right)^{2}\right] \\
& +(n-1) \int_{B_{r}^{\prime}}\left[\lambda^{+} \chi_{\{u>0\}}+\lambda^{-} \chi_{\{u<0\}}\right] \eta_{k}^{\prime} \\
& -\frac{1}{k} \int_{B_{r}^{\prime} \backslash B_{r-k}^{\prime}}\left|x^{\prime}\right|\left[\lambda^{+} \chi_{\{u>0\}}+\lambda^{-} \chi_{\{u<0\}}\right] .
\end{aligned}
$$


Letting $k \rightarrow 0$ gives

$$
\begin{aligned}
& 0=(n-2) \int_{B_{r}}|\nabla u|^{2}-r \int_{\partial B_{r}}|\nabla u|^{2}-2\left(u_{v}\right)^{2} \\
&+(n-1) \int_{B_{r}^{\prime}} \lambda^{+} \chi_{\{u>0\}}+\lambda^{-} \chi_{\{u<0\}}-r \int_{\partial B_{r}^{\prime}} \lambda^{+} \chi_{\{u>0\}}+\lambda^{-} \chi_{\{u<0\}} \\
&=(n-1) \int_{B_{r}}|\nabla u|^{2}-r \int_{\partial B_{r}}|\nabla u|^{2} \\
&+(n-1) \int_{B_{r}^{\prime}} \lambda^{+} \chi_{\{u>0\}}+\lambda^{-} \chi_{\{u<0\}}-r \int_{\partial B_{r}^{\prime}} \lambda^{+} \chi_{\{u>0\}}+\lambda^{-} \chi_{\{u<0\}} \\
&-\int_{B_{r}}|\nabla u|^{2}+2 r \int_{\partial B_{r}} u_{v}^{2} .
\end{aligned}
$$

Now, for smooth $\psi$

$$
\int_{B_{r}} u \Delta \psi+\int_{B_{r}} \nabla \psi \cdot \nabla u=\int_{\partial B_{r}} u \psi_{\nu} .
$$

We let $\psi$ to be a standard mollification of $u$, i.e., $\psi=\eta_{\epsilon} * u$, and let $\epsilon \rightarrow 0$ to obtain

$$
\int_{B_{r}} u \Delta u+\int_{B_{r}}|\nabla u|^{2}=\int_{\partial B_{r}} u u_{\nu} .
$$

Next, as a measure, $u \Delta u=0$, hence

$$
\begin{aligned}
0=(n-1) & \int_{B_{r}}|\nabla u|^{2}-r \int_{\partial B_{r}}|\nabla u|^{2} \\
& +(n-1) \int_{B_{r}^{\prime}} \lambda^{+} \chi_{\{u>0\}}+\lambda^{-} \chi_{\{u<0\}}-r \int_{\partial B_{r}^{\prime}} \lambda^{+} \chi_{\{u>0\}}+\lambda^{-} \chi_{\{u<0\}} \\
& -\int_{\partial B_{r}} u u_{v}+2 r \int_{\partial B_{r}} u_{v}^{2} .
\end{aligned}
$$

Multiplying both sides of the equation by $-r^{-n}$, we obtain that for a.e. $r \in(0, R)$

$$
\begin{aligned}
0=\left[\frac{1}{r^{n-1}} \int_{B_{r}}|\nabla u|^{2}\right]^{\prime}+\left[\frac{1}{r^{n-1}} \int_{B_{r}^{\prime}} \lambda^{+} \chi_{\{u>0\}}+\lambda^{-} \chi_{\{u<0\}}\right]^{\prime} \\
-\left[\frac{1 / 2}{r^{n}} \int_{\partial B_{r}} u^{2}\right]^{\prime}-\frac{1}{r^{n-1}} \int_{\partial B_{r}}\left(\frac{u}{\sqrt{2} r}-\sqrt{2} u_{v}\right)^{2} .
\end{aligned}
$$

To finish the proof, we now note that $W$ is an absolutely continuous function of $r$ and by the computation above

$$
W^{\prime}(r, u)=\frac{1}{r^{n-1}} \int_{\partial B_{r}}\left(\frac{u}{\sqrt{2} r}-\sqrt{2} u_{\nu}\right)^{2},
$$

for a.e. $r \in(0, R)$. Thus, $W^{\prime} \geqslant 0$, and $W^{\prime}=0$ on the interval $r_{1}<r<r_{2}$ if and only if $u$ is homogeneous of degree $1 / 2$ on the ring $r_{1}<|x|<r_{2}$. 
When studying blow-ups, one of the first questions that one may ask is, "what type of solutions may arise as blow-ups?" It is immediate that blow-ups are defined and minimizers in all of $\mathbb{R}^{n}$; that is they are minimizers in all compact subsets of $\mathbb{R}^{n}$. One of the most important uses of Weiss-type monotonicity formulas is the ability to prove the following result about all blow-ups.

COROLLARY 8.4 If $u_{0}$ is any blow-up, then $u_{0}$ is homogeneous of degree $1 / 2$.

Proof. By Theorem 4.2, if $u_{r} \rightarrow u_{0}$, over a subsequence $r=r_{k} \rightarrow 0$ then

$$
\int_{B_{1}}\left|\nabla u_{0}\right|^{2}=\lim _{r=r_{k} \rightarrow 0} \int_{B_{1}}\left|\nabla u_{r}\right|^{2} .
$$

Also, by Theorem 5.9,

$$
\chi_{\left\{ \pm u_{r_{k}}>0\right\}} \rightarrow \chi_{\left\{ \pm u_{0}>0\right\}} \quad \mathcal{H}^{n-1}-\text { a.e. on } \mathbb{R}^{n-1}
$$

and therefore

$$
\int_{B_{1}^{\prime}} \chi_{\left\{ \pm u_{0}>0\right\}}=\lim _{r=r_{k} \rightarrow 0} \int_{B_{1}^{\prime}} \chi_{\left\{ \pm u_{r}>0\right\}}
$$

Then for any $\rho>0$,

$$
W\left(\rho, u_{0}\right)=\lim _{r=r_{k} \rightarrow 0} W\left(\rho, u_{r}\right)=\lim _{r=r_{k} \rightarrow 0} W(\rho r, u)=W(0+, u),
$$

where we have used the strong convergence of $u_{r_{k}}$ to $u_{0}$ in $B_{\rho}$, see Theorem 4.2. Thus, $W\left(r, u_{0}\right)$ is constant; consequently, $u_{0}$ is homogeneous of degree $1 / 2$.

We conclude this section with the following simplified form of the Weiss energy functional for homogeneous minimizers.

LEMMA 8.5 If $u$ is a homogeneous of degree $1 / 2$ minimizer, then

$$
W(r, u) \equiv \lambda^{+} \mathcal{H}^{n-1}\left(\{u>0\} \cap B_{1}^{\prime}\right)+\lambda^{-} \mathcal{H}^{n-1}\left(\{u<0\} \cap B_{1}^{\prime}\right) .
$$

Proof. Because of the homogeneity, the formula is equivalent to

$$
W(r, u)=\frac{1}{r^{n-1}} \int_{B_{r}^{\prime}} \lambda^{+} \chi_{\{u>0\}}+\lambda^{-} \chi_{\{u<0\}} .
$$

This means that we have to show that the other two terms in the Weiss energy functional cancel each other; i.e.,

$$
\frac{1}{r^{n-1}} \int_{B_{r}}|\nabla u|^{2}=\frac{1 / 2}{r^{n}} \int_{\partial B_{r}} u^{2}
$$

Essentially, this has already been established in the proof of Theorem 8.1. Indeed, we have shown there that

$$
\int_{B_{r}}|\nabla u|^{2}=\int_{\partial B_{r}} u u_{v}
$$

as a consequence of the equality $u \Delta u=0$ in the sense of measures. Observing now that $r u_{v}=$ $(1 / 2) u$ on $\partial B_{r}$ for homogeneous of degree $1 / 2$ functions, we complete the proof. 


\section{Regular points}

We resume the study of nonnegative minimizers as described in the beginning of Section 7 .

In this section, our main focus will be on so-called regular free boundary points. Intuitively, we would like to say that a free boundary point is regular (or differentiable) if there exist a blow-up with a flat free boundary. To give formal definitions, we start with a special global minimizer, which we will call the half-plane solution.

THEOREM 9.1 (Half-plane solutions) The function

$$
\hat{u}(x, y)=\sqrt{2 / \pi} \operatorname{Re} \sqrt{x+i y}=\sqrt{\frac{x+\sqrt{x^{2}+y^{2}}}{\pi}}=\sqrt{2 / \pi} r^{1 / 2} \cos (\theta / 2)
$$

is a minimizer on any $U \Subset \mathbb{R}^{2}$. (Here $(r, \theta)$ are the polar coordinates in $\mathbb{R}^{2}$.) Similarly,

$$
\hat{u}\left(x_{1}, \ldots, x_{n}\right)=\sqrt{2 / \pi} \operatorname{Re} \sqrt{x_{n-1}+i x_{n}}
$$

is a minimizer on any $U \Subset \mathbb{R}^{n}, n \geqslant 2$. We call these minimizers half-plane solutions.

Proof. Blow-ups are minimizers and homogeneous of degree 1/2. To show the first part of the theorem, we note that the only harmonic functions in $\mathbb{R}_{ \pm}^{2}$ that are homogeneous of degree $1 / 2$ and nonnegative on $\mathbb{R} \times\{0\}$ have the form $\hat{u}=c \operatorname{Re} \sqrt{x+i y}$ (up to a reflection in $x$ ) for some constant $c$. Thus, we only need to identify the constant $c$. This will be accomplished by using variational techniques similar to the proof of Theorem 8.1. This time we choose $\tau_{\epsilon}(x, y)=(x, y)+\epsilon \eta(x, y)$ where $\eta(x, y)=(\gamma(x) \psi(y), 0)$. Now following the same ideas as in [1] and the proof of Theorem 8.1, we obtain

$$
\int_{\{\hat{u}>0\}} \operatorname{div}\left(|\nabla \hat{u}|^{2} \eta-2(\eta \cdot \nabla \hat{u}) \nabla \hat{u}\right)=-\int_{(\mathbb{R} \times\{y=0\}) \cap\{\hat{u}>0\}} \psi(0) \operatorname{div} \gamma .
$$

Now let $\psi(y) \equiv 1$ and $\gamma(x) \equiv 1$ on $(-\delta, \delta)$ for some small $\delta>0$. Then the right-hand side in (9.1) is equal to 1 by the fundamental theorem of calculus. For the left-hand side we approximate by isolating the origin

$$
\int_{\{\hat{u}>0\}} \operatorname{div}\left(|\nabla \hat{u}|^{2} \eta-2(\eta \cdot \nabla \hat{u}) \nabla \hat{u}\right)=\lim _{\epsilon \rightarrow 0} \int_{\{\hat{u}>0\} \backslash B_{\epsilon}} \operatorname{div}\left(|\nabla \hat{u}|^{2} \eta-2(\eta \cdot \nabla \hat{u}) \nabla \hat{u}\right) .
$$

We next want to apply the divergence theorem. For our specific $\hat{u}$ we have that on the zero set $\{\hat{u}=0\}$ :

$$
\eta \cdot v=(\gamma \psi, 0) \cdot(0, \pm 1)=0, \quad \eta \cdot \nabla \hat{u}=(\gamma \psi, 0) \cdot\left(0, \hat{u}_{y}\right)=0
$$

and therefore

$$
\lim _{\epsilon \rightarrow 0} \int_{\{\hat{u}>0\} \backslash B_{\epsilon}} \operatorname{div}\left(|\nabla \hat{u}|^{2} \eta-2(\eta \cdot \nabla \hat{u}) \nabla \hat{u}\right)=\lim _{\epsilon \rightarrow 0} \int_{\partial B_{\epsilon}}-|\nabla \hat{u}|^{2} \frac{x}{\epsilon}-2 \hat{u}_{x} \hat{u}_{\nu} .
$$

Explicit computations for $\hat{u}=c \operatorname{Re} \sqrt{x+i y}$ show that (in polar coordinates)

$$
|\nabla \hat{u}|^{2}=\frac{c^{2}}{4 r} \quad \text { and } \quad \hat{u}_{x}=\hat{u}_{v}=c \sqrt{\frac{\cos (\theta)+1}{8 r}} .
$$


Then

$$
\begin{aligned}
\lim _{\epsilon \rightarrow 0} \int_{\partial B_{\epsilon}}-|\nabla \hat{u}|^{2} \frac{x}{\epsilon}-2 \hat{u}_{x} \hat{u}_{v} & =c^{2} \int_{\partial B_{\epsilon}} \frac{-\cos (\theta)}{4 \epsilon}+2 \frac{\cos (\theta)+1}{8 r} \\
& =\frac{c^{2}}{4} \int_{\partial B_{1}} 1 \\
& =c^{2} \frac{\pi}{2} .
\end{aligned}
$$

Thus, $c^{2} \pi / 2=1$, and consequently $c=\sqrt{2 / \pi}$. This establishes the first part of the theorem.

For the second part of the theorem, we just observe that it is fairly straightforward to show that any minimizer $u$ in dimension $k \leqslant n$ can be "lifted" to a minimizer $\tilde{u}$ in dimension $n$ by $\tilde{u}\left(x_{1}, \ldots, x_{n}\right)=u\left(x_{n-k+1}, \ldots, x_{n}\right)$.

Definition 9.2 (Regular points). Let $u$ be a minimizer and $x \in \Gamma$. We call $x$ a regular point if there exists a blow-up $u_{0}$ of $u$ at $x_{0}$ such that $u_{0}$ is a rotation (in the first $n-1$ variables) of the half-plane solution $\hat{u}$.

The next theorem will give a characterization of regular points in terms of the so-called Weiss energy.

Definition 9.3 (Weiss energy at a point). For $x_{0} \in \Gamma_{u}$, the Weiss energy of $u$ at $x_{0}$ is defined as the limit $W\left(0+, u, x_{0}\right)$. We recall here that if $u_{0}$ is any blow-up of $u$ at the origin, then

$$
W\left(0+, u, x_{0}\right)=W\left(r, u_{0}\right),
$$

see the proof of Corollary 8.4. Moreover, since $u_{0}$ is homogeneous of degree $1 / 2$, by Lemma 8.5 we have that

$$
W\left(0+, u, x_{0}\right)=\mathcal{H}^{n-1}\left(\left\{u_{0}>0\right\} \cap B_{1}^{\prime}\right) .
$$

In particular, if $x_{0}$ is a regular point, then

$$
W\left(0+, u, x_{0}\right)=\mathcal{H}^{n-1}\left(\{\hat{u}>0\} \cap B_{1}^{\prime}\right)=\alpha_{n-1} / 2,
$$

where

$$
\alpha_{n-1}:=\mathcal{H}^{n-1}\left(B_{1}^{\prime}\right) .
$$

THEOREM 9.4 (Weiss energy and regular points) Let $u$ be a minimizer and $x_{0} \in \Gamma_{u}$. Then

$$
W\left(0+, u, x_{0}\right) \geqslant \alpha_{n-1} / 2
$$

and equality holds if and only if $x_{0}$ is a regular point.

To prove Theorem 9.4 we will use the following lemma.

Lemma 9.5 Let $f: S^{n-1} \rightarrow \mathbb{R}$ be continuous on $S^{n-1}$ and real analytic in $S^{n-1} \backslash\left(S^{n-1}\right)^{\prime}$. By using spherical coordinates we may consider $f: \bar{R} \rightarrow \mathbb{R}$, where $R:=(-\pi, \pi) \times(0, \pi) \times \cdots \times(0, \pi)$ and $f$ is $2 \pi$-periodic in $\theta_{1}$; i.e.

$$
f\left(-\pi, \theta_{2}, \ldots, \theta_{n-1}\right)=f\left(\pi, \theta_{2}, \ldots, \theta_{n-1}\right) .
$$


If we Steiner symmetrize $f$ in $\theta_{1}$-variable to obtain $\tilde{f}$, then

$$
\begin{gathered}
\int_{S^{n-1}}\left|\nabla_{\theta} \tilde{f}\right|^{2} \leqslant \int_{S^{n-1}}\left|\nabla_{\theta} f\right|^{2}, \\
\int_{S^{n-1}}|\tilde{f}|^{2}=\int_{S^{n-1}}|f|^{2} .
\end{gathered}
$$

Equality holds in (9.2) if and only if $f=\tilde{f}$ modulo translation in $\theta_{1}$-variable.

The latter means that for some constant $c$

$$
f\left(\theta_{1}, \theta_{2}, \ldots, \theta_{n-1}\right) \equiv \tilde{f}\left(\theta_{1}+c, \theta_{2}, \ldots, \theta_{n-1}\right) .
$$

Proof. The spherical gradient of $f$ on $S^{n-1}$ in local coordinates is given by

$$
\nabla_{\theta} f=\left(\frac{f_{\theta_{i}}}{\sin \theta_{n-1} \cdots \sin \theta_{i+1}}\right)
$$

so that

$$
\left|\nabla_{\theta} f\right|^{2}=\sum_{i=1}^{n-1} \frac{f_{\theta_{i}}^{2}}{\sin ^{2} \theta_{n-1} \cdots \sin ^{2} \theta_{i+1}}
$$

We now define

$$
R_{\epsilon}:=\left\{\theta \in R \mid \theta_{2}, \ldots, \theta_{n-2} \in(\epsilon, \pi-\epsilon), \quad \theta_{n-1} \in(\epsilon, \pi / 2-\epsilon) \cup(\pi / 2+\epsilon, \pi-\epsilon)\right\}
$$

and observe that

$$
\frac{\sin ^{n-2} \theta_{n-1} \ldots \sin \theta_{2}}{\sin ^{2} \theta_{n-1} \cdots \sin ^{2} \theta_{i+1}} \text { and } \sin ^{n-2} \theta_{n-1} \ldots \sin \theta_{2}
$$

are positive and continuous on $R_{\epsilon}$ and independent of $\theta_{1}$ and that $f$ real analytic in $R_{\epsilon}$. Besides, $f$ is $2 \pi$-periodic in $\theta_{1}$-variable; i.e., $f\left(-\pi, \theta_{2}, \ldots, \theta_{n-1}\right)=f\left(\pi, \theta_{2}, \ldots, \theta_{n-1}\right)$. If we Steiner symmetrize in $\theta_{1}$-variable to obtain $\tilde{f}$, then by [10, Theorem 2.31 ]

$$
\begin{aligned}
\sum_{i=1}^{n-1} \int_{R_{\epsilon}} \frac{\tilde{f}_{\theta_{i}}^{2}}{\sin ^{2} \theta_{n-1} \ldots \sin ^{2} \theta_{i+1}} \sin ^{n-2} \theta_{n-1} \ldots \sin \theta_{2} \\
\quad \leqslant \sum_{i=1}^{n-1} \int_{R} \frac{f_{\theta_{i}}^{2}}{\sin ^{2} \theta_{n-1} \ldots \sin ^{2} \theta_{i+1}} \sin ^{n-2} \theta_{n-1} \ldots \sin \theta_{2}
\end{aligned}
$$

and

$$
\int_{R_{\epsilon}} \tilde{f}^{2} \sin ^{n-2} \theta_{n-1} \ldots \sin \theta_{2}=\int_{R_{\epsilon}} f^{2} \sin ^{n-2} \theta_{n-1} \ldots \sin \theta_{2}
$$

and equality holds in (9.6) if and only if $f=\tilde{f}$ modulo translations in $\theta_{1}$-variable. already Steiner symmetric in $\theta_{1}$-variable. We then let $\epsilon \rightarrow 0$ to obtain (9.2). And again, the equality in (9.2) will hold if and only if $f=\tilde{f}$ modulo translations in $\theta_{1}$-variable.

We may now prove Theorem 9.4. 
Proof. Let $u$ be a minimizer and $x_{0} \in \Gamma$. Let $v$ be a blow-up of $u$ at $x_{0}$. Since $v$ is homogeneous of degree $1 / 2$ and harmonic when positive, restricted to the unit sphere $S^{n-1}, v$ satisfies the differential equation

$$
-\Delta_{S^{n-1}} v=\lambda v \quad \text { in } \Sigma:=\{v>0\} \cap S^{n-1},
$$

where $\Delta_{S^{n-1}}$ is the spherical Laplacian and

$$
\lambda=\frac{1}{2}\left(n-2+\frac{1}{2}\right)=\frac{2 n-3}{4} .
$$

Since $v$ is nonnegative, we conclude that $v$ is a principal eigenfunction of $-\Delta_{S^{n-1}}$ associated with $\Sigma$. We now consider $v$ in the place of $f$ in Lemma 9.5 and Steiner symmetrize it in $\theta_{1}$-variable to obtain $\tilde{v}$. Then,

$$
\frac{\int_{S^{n-1}}|\nabla \tilde{v}|^{2}}{\int_{S^{n-1}} \tilde{v}^{2}} \leqslant \frac{\int_{S^{n-1}}|\nabla v|^{2}}{\int_{S^{n-1}} v^{2}} .
$$

If $\tilde{\Sigma}$ is the Steiner symmetrization of $\Sigma$, then we can replace $S^{n-1}$ in (9.8) by $\tilde{\Sigma}$ and $\Sigma$ respectively, implying that the principal eigenvalue $\tilde{\lambda}$ of $\tilde{\Sigma}$ satisfies

$$
\tilde{\lambda} \leqslant \lambda .
$$

Moreover, the equality is achieved if and only if $v=\tilde{v}$ (and $\tilde{\Sigma}=\Sigma$ ) modulo translations in $\theta_{1^{-}}$variable. We next note that we have a freedom in choosing the $\theta_{1}$-variable. Namely, if we make an orthogonal rotation in $\mathbb{R}^{n-1}$ and leave $x_{n}$ unchanged, we may reintroduce the spherical coordinates and reapply the Steiner symmetrization in $\theta_{1}$. By a limiting procedure (similar to the Appendix in [4]) we thus construct a set $\Sigma^{*} \subset S^{n-1}$, whose complement is a thin spherical cap; i.e.

$$
\Sigma^{*}=S^{n-1} \backslash C_{t}, \quad C_{t}:=\left\{\left(x^{\prime}, 0\right) \in S^{n-1} \mid x_{n-1} \leqslant t\right\},
$$

for some $|t|<1$, with the corresponding symmetrized function $v^{*}$ on $S^{n-1}$ such that $\left\{v^{*}>0\right\}=$ $\Sigma^{*}$. Moreover, we will have that

$$
\mathcal{H}^{n-2}\left(\left\{v^{*}>0\right\} \cap S^{n-1}\right)=\mathcal{H}^{n-2}\left(\{v>0\} \cap S^{n-1}\right)
$$

and that the analogue of (9.8) holds with $v^{*}$ in place of $\tilde{v}$. Hence, if $\lambda^{*}$ is the principal eigenvalue of $\Sigma^{*}$, then

$$
\lambda^{*} \leqslant \lambda
$$

Next, by considering the half-plane solution $\hat{u}$, we observe that $\lambda$ is the principal eigenvalue of $\hat{\Sigma}=S^{n-1} \backslash C_{0}=\{\hat{u}>0\} \cap S^{n-1}$. This immediately implies that $t \leqslant 0$ in the representation (9.9), since strictly smaller domains have strictly larger principal eigenvalues. This enables us to conclude that

$$
\begin{aligned}
W(v, r) & =(n-1) \mathcal{H}^{n-2}\left(\{v>0\} \cap\left(S^{n-1}\right)^{\prime}\right) \\
& =(n-1) \mathcal{H}^{n-2}\left(\left\{v^{*}>0\right\} \cap\left(S^{n-1}\right)^{\prime}\right) \geqslant \alpha_{n-1} / 2 .
\end{aligned}
$$

If equality above is achieved, then we must have $\lambda^{*}=\lambda$ and that $v^{*}$ is a multiple of the half-plane solution $\hat{u}$ (by the uniqueness of the principal eigenvalue). But then we must also have equality in (9.8) for any Steiner symmetrization $\tilde{v}$. Recall that this implies that $v=\tilde{v}$ modulo a rotation in $\mathbb{R}^{n-1} \times\{0\}$. As a consequence, we obtain that $v=v^{*}=c \hat{u}$. Since the constant $c$ is uniquely determined (see the proof of Theorem 9.1) we conclude that $v$ is a rotation of the half-plane solution and that $x_{0}$ is a regular point. This concludes the proof. 
One of the main difficulties in studying blow-ups is that a priori it is not clear if different subsequences converge to different blow-up solutions; i.e., if $r_{k} \rightarrow 0$ and $r_{k}^{\prime} \rightarrow 0$ are two different subsequences and $u_{r_{k}} \rightarrow u_{0}$ and $u_{r_{k}^{\prime}} \rightarrow u_{0}^{\prime}$, is it true that $u_{0} \equiv u_{0}^{\prime}$ ? At this stage we give only a partial answer to this question, by noticing that at regular points the Weiss energy equals $\alpha_{n-1} / 2$. Applying Theorem 9.4, we then obtain the following.

COROLlary 9.6 If $x_{0} \in \Gamma$ and $x_{0}$ is a regular point, then all blow-ups at $x_{0}$ are rotations of the half-plane solution.

Note that it is not immediately clear that the blow-ups have to coincide. They could, in principle, be rotations of each other. In Section 10 we show that this is not the case, at least in dimension $n=3$.

The next theorem will be useful in Section 10. We present the proof here, since the ideas are similar to those in the proof of Theorem 9.4.

THEOREM 9.7 Let $u$ be a minimizer in dimension $n=3$ and $0 \in \Gamma$. Suppose that in a small enough thin ball $B_{\rho}^{\prime}$, the positivity set of $u$ lies above a graph in $x_{1}$ direction; i.e.

$$
\{u>0\} \cap B_{\rho}^{\prime}=\left\{x_{1}>\phi\left(x_{2}\right)\right\} \cap B_{\rho}^{\prime},
$$

for some function $\phi$. Then every free boundary point of $u$ in $B_{\rho}^{\prime}$ is a regular point.

We will need the following lemma for the proof of Theorem 9.7.

LEMma 9.8 Assume the same hypotheses as given in Theorem 9.7. If $x_{0} \in \Gamma \cap B_{\rho}^{\prime}$, and $v$ is a blow-up of $u$ at $x_{0}$, then the zero set of $v$ consists of a single connected cone.

The zero set of a blow-up is always connected topologically (because of the homogeneity). When we say that the zero set consists of a single connected cone, we mean that the interior $\{v=0\}^{\circ}$ (in the topology of $\mathbb{R}^{n-1} \times\{0\}$ ) is connected.

Proof. Since blow-ups are homogeneous, it is clear that the zero set of $v$ is a cone. Let $y=$ $\left(y_{1}, y_{2}, 0\right) \in\{v=0\}^{\circ}$. We claim that

$$
v\left(t, y_{2}, 0\right)=0 \text { for all } t \leqslant y_{1} \text {. }
$$

By the nondegeneracy in the thin space (Theorem 5.5), we know that for large enough $k, u_{r_{k}}=0$ in a small thin ball around $\left(y_{1}, y_{2}, 0\right)$. Then, from the assumption on $u$ we have that $u_{r_{k}}\left(t, y_{2}, 0\right)=0$ for $t \leqslant y_{1}$ and consequently $v\left(t, y_{2}, 0\right)=0$. This geometric property, along with the fact that the zero set of $v$ is a cone, proves the lemma.

Proof of Theorem 9.7. Let $x_{0} \in \Gamma \cap B_{\rho}$ and $v$ be a blow-up of $u$ at $x_{0}$. Then $v$ is homogeneous of degree $1 / 2$ and harmonic off the zero set. We also obtain on $S^{2}$

$$
-\Delta_{S^{2}} v=3 / 4 v
$$

Here $3 / 4=\lambda$ is the dimensional constant that is a result of $v$ being homogeneous of degree $1 / 2$. Since the free boundary of $u$ can be represented as a graph, by Lemma 9.8 we know that the zero set of $v$ consists of a single connected cone. $v$, which is nonnegative, must then be the principal eigenfunction on $S^{2}$ associated with its coincidence set. We now utilize the fact that our minimizer is in dimension three. Specifically, $\{v=0\} \cap\left(S^{2}\right)^{\prime}$ must be a thin spherical cap (since it is a 
1-dimensional curve). Since the principal eigenvalue of $v$ is fixed at $3 / 4$, this is possible only if $\{v=0\} \cap\left(S^{2}\right)^{\prime}$ is a half-thin-sphere. Then

$$
\mathcal{H}^{2}(\{v>0\})=\alpha_{2} / 2,
$$

and by Theorem 9.4, we conclude that $x_{0}$ is a regular point.

\section{Regularity of the free boundary in dimension three}

In this section we prove that in three dimensions the free boundary is locally a $C^{1}$ graph near regular points. Here again we consider only nonnegative minimizers, as described in the beginning of Section 7.

THEOREM 10.1 Let $u$ be a minimizer in dimension $n=3$. Then the set of regular points is dense and relatively open in $\Gamma$. Furthermore, the set of regular points is locally a $C^{1}$ graph in $\mathbb{R}^{2} \times\{0\}$.

Remark 10.2. We would like to observe here that in dimension $n=2$ the free boundary has a very simple structure: it is discrete. Indeed, assuming that $x_{0} \in \Gamma$ is a limit point of some $x_{k} \in \Gamma$, $x_{k} \neq x_{0}$, consider the limit $u_{0}$ of the rescalings $u_{r}(x)=u\left(x_{0}+r x\right) / r^{1 / 2}$ with $r=r_{k}=\left|x_{k}-x_{0}\right|$, over a subsequence. We may also assume that $\left(x_{k}-x_{0}\right) / r_{k} \rightarrow \xi_{0} \in \partial B_{1}^{\prime}$. Then, on one hand, $u_{0}$ must be homogeneous of degree $1 / 2$ (by Corollary 8.4) and thus have the form $u_{0}=\hat{u}$ (up to reflection in $x$ ), and on the other hand, we must have $\xi_{0} \in \Gamma_{u_{0}}$. However, $\Gamma_{u_{0}}=\{0\}$, which is a contradiction. Thus, the free boundary $\Gamma$ is discrete in dimension $n=2$.

To prove Theorem 10.1, we start with a technical lemma (that works in any dimension $n \geqslant 2$ ) on the growth of minimizers away from the plane $\mathbb{R}^{n-1} \times\{0\}$.

LEMmA 10.3 (Linear growth) Let $u$ be a minimizer in $B_{1}$ with $\|u\|_{C^{1 / 2}\left(B_{1}\right)} \leqslant M$, and let $\epsilon>0$. There exists a constant $c=c_{n, M}>0$ depending only on $n$ and $M$ such that if $x^{\prime} \in B_{1 / 2}^{\prime}$, $\operatorname{dist}\left(x^{\prime}, \Gamma\right) \leqslant \epsilon$ and $\left|x_{n}\right| \leqslant \epsilon$, then

$$
u\left(x^{\prime}, x_{n}\right) \geqslant \frac{c\left|x_{n}\right|}{\sqrt{\epsilon}} .
$$

Proof. Throughout this proof $c$ and $C$ will be constants depending only on dimension and on $M$. Also, without loss of generality, we may additionally assume $x_{n} \geqslant 0$. We consider the three cases:

$$
x^{\prime} \in\{u=0\} \backslash \Gamma, \quad x^{\prime} \in \Gamma, \quad u\left(x^{\prime}, 0\right)>0 .
$$

If $x^{\prime} \in \Gamma$, then we may use a compactness argument similar to the one used in Lemma 7.2, to conclude:

$$
u\left(x^{\prime}, x_{n}\right) \geqslant c \sqrt{x_{n}}=\frac{c x_{n}}{\sqrt{x_{n}}} \quad \text { in } B_{1}\left(x^{\prime}, 0\right) .
$$

In particular,

$$
u\left(x^{\prime}, x_{n}\right) \geqslant \frac{c x_{n}}{\sqrt{\epsilon}} \text { for } x_{n}<\epsilon .
$$

If $x^{\prime} \notin \Gamma$, then let $\operatorname{dist}\left(x^{\prime}, \Gamma\right)=\delta \leqslant \epsilon$. If $x^{\prime} \in\{u=0\}$, then by Lemma 7.2

$$
\frac{c}{\sqrt{\operatorname{dist}\left(x^{\prime}, \Gamma\right)}} \leqslant \frac{\partial u}{\partial x_{n}}\left(x^{\prime}, 0\right) \leqslant \frac{C}{\sqrt{\operatorname{dist}\left(x^{\prime}, \Gamma\right)}} .
$$


Then we may compare $u$ to the harmonic function $f(x)=x_{n}$, and by the boundary Harnack principle [9],

$$
\frac{c x_{n}}{\sqrt{2 \epsilon}} \leqslant u\left(x^{\prime}, x_{n}\right) \quad \text { in } B_{\delta / 2}\left(x^{\prime}, 0\right) .
$$

If $x_{n}>\delta / 2$, then let $y^{\prime}$ be the closest point on the free boundary to $x^{\prime}$. As stated earlier,

$$
u\left(y^{\prime}, y_{n}\right) \geqslant \frac{c y_{n}}{\sqrt{y_{n}}} .
$$

For $t>\delta$, we can connect $\left(y^{\prime}, t\right)$ to $\left(x^{\prime}, t\right)$ by five balls of radius $\delta / 4$. Then, by using the Harnack inequality on this Harnack chain, we obtain that for $\delta / 2<t \leqslant \epsilon$ :

$$
u\left(x^{\prime}, t\right) \geqslant \frac{c^{5} t}{\sqrt{t}} \geqslant \frac{c^{5} t}{\sqrt{\epsilon}} .
$$

We now consider the last case when $u\left(x^{\prime}, 0\right)>0$. For $\delta<t \leqslant \epsilon$ we may again use a Harnack chain and obtain as in equation (10.2)

$$
u\left(x^{\prime}, t\right) \geqslant \frac{c t}{\sqrt{\delta}} \geqslant \frac{c t}{\sqrt{\epsilon}} .
$$

But now, since $u$ is harmonic in the tube $B_{\delta}^{\prime} \times(-\epsilon, \epsilon)$, we may continue a chain of three more Harnack balls of radius $\delta / 2$, and we obtain for $0 \leqslant t \leqslant \delta$

$$
u\left(x^{\prime}, t\right) \geqslant \frac{c^{3} \delta}{\sqrt{\delta}} \geqslant \frac{c^{3} t}{\sqrt{\epsilon}} .
$$

This implies the claimed estimate.

For the remainder of this section we will be working in dimension three, so the free boundary will be contained in $\mathbb{R}^{2} \times\{0\}$. Accordingly, our functional $J$ will be

$$
J(v)=\int_{\mathbb{R}^{3}}|\nabla v|^{2} d x+\int_{\mathbb{R}^{2} \times\{0\}} \chi_{\left\{v\left(x_{1}, x_{2}, 0\right)>0\right\}} d \mathcal{H}^{2}
$$

and our half-plane solution will be

$$
\hat{u}(x)=\sqrt{2 / \pi} \operatorname{Re} \sqrt{x_{2}+i x_{3}} .
$$

To prove that the free boundary is differentiable we will use the Alexandrov Reflection technique, adapted to our problem.

For $R, h>0$ consider the tube (cylinder)

$$
T_{R, h}:=B_{R}^{\prime} \times(-h, h)
$$

and for every $\omega \in[0,2 \pi)$ let

$$
v_{\omega}:=(-\sin \omega, \cos \omega, 0), \quad \Pi_{\omega}:=\left\{x \cdot v_{\omega}=0\right\} .
$$

Note that $\Pi_{\omega}$ is the plane spanned by the vectors $(\cos \omega, \sin \omega, 0)$ and $(0,0,1)$. For a point $x \in T_{R, h}$ consider the reflection $\bar{x}_{\omega}$ with respect to $\Pi_{\omega}$. Using polar coordinates in $\mathbb{R}^{2}$, if $r \geqslant 0$ and $\theta$ are such that

$$
x=\left(r \cos (\omega+\theta), r \sin (\omega+\theta), x_{3}\right),
$$


then

$$
\bar{x}_{\omega}=\left(r \cos (\omega-\theta), r \sin (\omega-\theta), x_{3}\right) .
$$

For a given function $v$ on $T_{R, h}$, to compare $v$ and its reflection with respect to $\Pi_{\omega}$, we introduce the quantity

$$
\begin{aligned}
\phi_{\omega}\left(r, \theta, x_{3} ; v\right):= & v(x)-v\left(\bar{x}_{\omega}\right) \\
= & v\left(r \cos (\omega+\theta), r \sin (\omega+\theta), x_{3}\right) \\
& \quad-v\left(r \cos (\omega-\theta), r \sin (\omega-\theta), x_{3}\right) .
\end{aligned}
$$

LEMma 10.4 (Reflection principle) Let $u$ be a minimizer in the tube $T_{R, h}$ such that for every $y \in \partial T_{R, h}$ with $y \cdot v_{\omega} \geqslant 0$, we have

$$
u(y) \geqslant u\left(\bar{y}_{\omega}\right) .
$$

Also, suppose that there exists at least one point $z \in \partial T_{R, h}$ such that $z \cdot v_{\omega}>0$ and

$$
u(z)>u\left(\bar{z}_{\omega}\right) .
$$

Then for every $x \in T_{R, h}$ with $x \cdot v_{\omega} \geqslant 0$ we have

$$
u(x) \geqslant u\left(\bar{x}_{\omega}\right) .
$$

In terms of the function $\phi$, this lemma says that to verify the inequality

$$
\phi\left(r, \theta, x_{3} ; u\right) \geqslant 0, \quad \text { for all }\left(r, \theta, x_{3}\right) \in[0, R] \times(0, \pi) \times[-h, h],
$$

it is enough to do it only when $r=R$ or $\left|x_{3}\right|=h$, provided a strict inequality holds at least at one of such points.

Proof. Define $\tilde{u}(y) \equiv u\left(\bar{y}_{\omega}\right)$. Then $u$ and $\tilde{u}$ are both minimizers in the set $U T_{R, h}=\left\{x \in T_{R, h} \mid\right.$ $\left.x \cdot v_{\omega} \geqslant 0\right\}$ (the upper half-tube in direction $v_{\omega}$ ). By Theorem 2.2 we obtain that $w=\max \{u, \tilde{u}\}$ is also a minimizer. In particular, both $w$ and $u$ are harmonic in $U T_{R, h}^{ \pm}=U T_{R, h} \cap \mathbb{R}_{ \pm}^{3}$. Moreover, by definition, we have that $w \geqslant u$ in $U T_{R, h}^{ \pm}$and $w=u$ on $\partial U T_{R, h}$. Now, the existence of the boundary point $z$ as in the statement of the lemma implies that $w \equiv u$ in a neighborhood of $z$. Suppose, for definiteness, that $z_{3} \geqslant 0$. Then, by the strong maximum principle we will have that $w \equiv u$ in $U T_{R, h}^{+}$. In turn, this implies that $w \equiv u$ on $\partial U T_{R, h}^{-}$as well, and consequently that $w \equiv u$ in $U T_{R, h}^{-}$. Thus, $w$ coincides with $u$ in all of $U T_{R, h}$, which is equivalent to having $\tilde{u} \leqslant u$ there.

For the next result, we consider slightly more general reflection with respect to shifted planes $y+\Pi_{\omega}$ for $y \in \mathbb{R}^{2} \times\{0\}$, namely

$$
\begin{aligned}
\phi_{\omega}^{y}\left(r, \theta, x_{3} ; v\right):= & v(y+x)-v\left(y+\bar{x}_{\omega}\right) \\
= & v\left(y_{1}+r \cos (\omega+\theta), y_{2}+r \sin (\omega+\theta), x_{3}\right) \\
& \quad-v\left(y_{1}+r \cos (\omega-\theta), y_{2}+r \sin (\omega-\theta), x_{3}\right) .
\end{aligned}
$$

Typically, we will let $y$ vary in a narrow thin strip

$$
S_{\epsilon}:=(-1,1) \times(-\epsilon, \epsilon) \times\{0\} .
$$


THEOREM 10.5 Let $\left\{u_{k}\right\}$ be a sequence of minimizers in the ball $B_{4}$ converging to the half-plane solution $\hat{u}(x)=\sqrt{2 / \pi} \operatorname{Re} \sqrt{x_{2}+i x_{3}}$. Then for every $\delta>0$, there exists $\epsilon=\epsilon_{\delta}$ and $k_{\delta}$, such that for $y \in S_{\epsilon},\left|x_{3}\right| \leqslant 1,0<r \leqslant 1, \theta \in(0, \pi)$ we have

$$
\phi_{\omega}^{y}\left(r, \theta, x_{3} ; u_{k}\right) \begin{cases}\geqslant 0, & \omega \in\left(\delta, \frac{\pi}{2}-\delta\right) \cup\left(\frac{3 \pi}{2}+\delta, 2 \pi-\delta\right), \\ \leqslant 0, & \omega \in\left(\frac{\pi}{2}+\delta, \pi-\delta\right) \cup\left(\pi+\delta, \frac{3 \pi}{2}-\delta\right),\end{cases}
$$

if $k \geqslant k_{\delta}$.

Proof. We only prove the theorem for the case $\omega \in(\delta, \pi / 2-\delta) \cup(3 \pi / 2+\delta, \pi-\delta)$, the other case being similar.

Let $\epsilon=\epsilon_{\delta}>0$ be a small constant to be specified due course. For $y \in S_{\epsilon}, x=(r \cos (\omega+$ $\left.\theta), r \sin (\omega+\theta), x_{3}\right)$ with $0<r \leqslant 1$ and $\theta \in(0, \pi)$, we denote

$$
z=y+x, \quad \bar{z}=y+\bar{x}_{\omega} .
$$

Then, we have to show that

$$
u_{k}(z) \geqslant u_{k}(\bar{z}) \text {. }
$$

In order to do so, we split $T_{2,2}$ into the following four regions

$$
\begin{aligned}
& U_{1}=T_{2, \epsilon} \cap\left\{x_{2}>2 \epsilon\right\}, \\
& U_{2}=T_{2, \epsilon} \cap\left\{\left|x_{2}\right| \leqslant 2 \epsilon\right\}, \\
& U_{3}=T_{2, \epsilon} \cap\left\{x_{2}<-2 \epsilon\right\}, \\
& U_{4}=T_{2,2} \cap\left\{\left|x_{3}\right| \geqslant \epsilon\right\}=T_{2,2} \backslash T_{2, \epsilon} .
\end{aligned}
$$

Let also

$$
\begin{gathered}
\tilde{U}_{1}=T_{2, \epsilon} \cap\left\{x_{2}>\delta / 2\right\}, \\
\tilde{U}_{3}=T_{2, \epsilon} \cap\left\{x_{2}<-\delta / 2\right\} .
\end{gathered}
$$

Note that $\tilde{U}_{1} \subset U_{1}$ and $\tilde{U}_{3} \subset U_{3}$, if $\epsilon<\delta / 4$.

(1) Suppose $z \in U_{4}$. Then, since $v_{\omega} \cdot(0,1,0)=\cos \omega \geqslant \sin \delta$, a direct computation shows that

$$
\partial_{v_{\omega}} \hat{u} \geqslant c_{\delta, \epsilon}>0 \quad \text { in } U_{4} .
$$

If $k$ is large enough so that $u_{k}-\hat{u}$ is small in $C^{1}$ norm in $U_{4}$, then

$$
\partial_{v_{\omega}} u_{k}>0 \text { in } U_{4}
$$

By noticing that $\bar{z}=z-2(\sin \theta) v_{\omega}$ we immediately obtain

$$
u_{k}(z)>u_{k}(\bar{z})
$$

(2) Suppose now $z \in T_{2,2} \backslash T_{2, \epsilon}$. We then note that it would be sufficient to consider the case $r=1$, i.e., to verify that

$$
\phi_{\omega}^{y}\left(1, \theta, x_{3} ; u_{k}\right) \geqslant 0 \quad \text { for }\left|x_{3}\right| \leqslant \epsilon .
$$


The general case $0<r \leqslant 1$ will follow then form the reflection principle in Lemma 10.4 applied to the tube $T_{1, \epsilon}$ and the shifted function $u(y+\cdot)$. We therefore fix $r=1$ in the rest of the proof. Then

$$
\begin{aligned}
& z=\left(y_{1}+\cos (\omega+\theta), y_{2}+\sin (\omega+\theta), x_{3}\right), \\
& \bar{z}=\left(y_{1}+\cos (\omega-\theta), y_{2}+\sin (\omega-\theta), x_{3}\right) .
\end{aligned}
$$

We will consider several subcases, depending on the location of points $z$ and $\bar{z}$. In this regard, we note that if $\omega \in(\delta, \pi / 2-\delta) \cup(3 \pi / 2+\delta, 2 \pi-\delta)$ and $\theta \in(0, \pi)$, then

$$
|\sin (\omega \pm \theta)| \leqslant|\sin \delta| \Rightarrow|\sin (\omega \mp \theta)|>|\sin \delta| .
$$

Hence, if we choose $\epsilon<(\sin \delta) / 4$, and use that $\left|y_{2}\right| \leqslant \epsilon$, then we have the following implications:

$$
\begin{array}{lll}
\left|\bar{z}_{2}\right| \leqslant 2 \epsilon & \Rightarrow \quad z_{2}>\delta / 2, \\
\left|z_{2}\right| \leqslant 2 \epsilon & \Rightarrow \quad \bar{z}_{2}<-\delta / 2 .
\end{array}
$$

Also note that we always have $z_{2} \geqslant \bar{z}_{2}$ in the considered ranges of $\omega$ and $\theta$.

Before proceeding, we note that by using the $C^{\alpha}$ convergence and the thin nondegeneracy as in the proof of Theorem 5.9, we may choose $k$ large enough so that

$$
\Gamma_{u_{k}} \cap T_{2,2} \subset\left\{\left|x_{2}\right|<\epsilon\right\} \cap B_{2}^{\prime}(0) .
$$

We then consider the following subcases.

(2a) $\left|\bar{z}_{2}\right| \leqslant 2 \epsilon$ and consequently $z_{2}>\delta / 2$. Let $\epsilon$ be so small that

$$
\sup _{U_{2}} u_{k} \leqslant C \sqrt{\epsilon} \leqslant \sqrt{\delta / 4 \pi}=\frac{1}{2} \inf _{\tilde{U}_{1}} \hat{u} .
$$

Then, using $L^{\infty}$ convergence of $u_{k}$ to $\hat{u}$ on $\tilde{U}_{1}$, we can guarantee that

$$
\sup _{U_{2}} u_{k} \leqslant \inf _{\tilde{U}_{1}} u_{k}
$$

if $k$ is large enough. This implies that $u_{k}(z) \geqslant u_{k}(\bar{z})$, which is equivalent to (10.5).

(2b) $\left|z_{2}\right| \leqslant 2 \epsilon$ and consequently $\bar{z}_{2}<-\delta / 2$. We can make $\epsilon$ even smaller to have

$$
\frac{c}{\sqrt{3 \epsilon}}\left|x_{3}\right| \geqslant 2 \hat{u}\left(x_{1}, x_{2}, x_{3}\right) \quad \text { for } x_{2} \leqslant-\delta / 2 \text {, and }\left|x_{3}\right| \leqslant \epsilon,
$$

for the constant $c$ as given in Lemma 10.3. Note that in this case we have $z \in U_{2}, \operatorname{dist}\left(z, \Gamma_{u_{k}}\right) \leqslant 3 \epsilon$ and $\bar{z} \in \tilde{U}_{3}$. Then by Lemma 10.3

$$
u_{k}(z) \geqslant 2 \hat{u}(\bar{z}) .
$$

For $x=\left(x^{\prime}, 0\right) \in \tilde{U}_{3}$, we know that $u_{k}\left(x^{\prime}, 0\right)=0$, for large $k$, and by the $C^{1}$ convergence of harmonic functions up to the set $\left\{x_{3}=0\right\}$ and we obtain that

$$
u_{k}(z) \geqslant u_{k}(\bar{z})
$$


Therefore inequality (10.5) also follows in this case.

(2c) $z_{2}>2 \epsilon$ and $\bar{z}_{2}<-2 \epsilon$. In this case, we may use $L^{\infty}$ convergence of $u_{k} \rightarrow \hat{u}$ again to obtain

$$
\sup _{U_{3}} u_{k} \leqslant \inf _{U_{1}} u_{k}
$$

which implies (10.5).

(2d) Both $z_{2}, \bar{z}_{2}>2 \epsilon$. Similar to the case (1), if $x \in U_{1}$, we may use $C^{1}$ convergence to obtain that

$$
\partial_{v_{\omega}} u_{k}>0 \text { in } U_{1}
$$

and consequently (10.5).

(2e) Both $z_{2}, \bar{z}_{2}<-2 \epsilon$. This is our only remaining concern. Without loss of generality we may further assume $x_{3} \geqslant 0$. On $U_{3}$ we know that $\partial_{x_{3}} \partial_{v_{\omega}} \hat{u}>c_{\delta, \epsilon}$. On $U_{3}$ we may use $C^{2}$ convergence up to the boundary $\left\{x_{3}=0\right\}$ to obtain that for large enough $k$

$$
\partial_{x_{3}} \partial_{v_{\omega}} u_{k}>0 \text { in } U_{3}
$$

Since $\partial_{v_{\omega}} u_{k}\left(x_{1}, x_{2}, 0\right)=0$ for $x_{2}<-\epsilon$, we obtain

$$
\partial_{v_{\omega}} u_{k}>0 \quad \text { in } U_{3}
$$

and consequently (10.5).

Thus, we have considered all cases, so we may conclude that inequality (10.5) is true for large enough $k$. This also completes the proof of the theorem.

The technical details of the previous proof would have been simpler if instead of letting $y \in S_{\epsilon}$, we had only considered $y=0$. The usefulness of allowing $y$ to vary is seen in the following corollary.

Corollary 10.6 Let $\left\{u_{k}\right\}, \hat{u}, \delta, \epsilon=\epsilon_{\delta}, k_{\delta}$ be as in Theorem 10.5. If $k \geqslant k_{\delta}$, then in the thin strip $S_{\epsilon}, u_{k}$ is monotone nondecreasing in the directions $e_{\omega}:=(\cos \omega, \sin \omega, 0)$ for $\omega \in$ $(\delta, \pi / 2-\delta) \cup(\pi / 2+\delta, \pi-\delta)$.

Proof. Let $x$ and $r>0$ be such that $x, x+r e_{\omega} \in S_{\epsilon}$. Pick $y$ to be the midpoint, so that $y=$ $x+(r / 2) e_{\omega}$. Then, noticing that $e_{\omega}=v_{\omega-\pi / 2}$, by Theorem 10.5 we obtain:

$$
\begin{aligned}
u_{k}\left(x+r e_{\omega}\right) & =u_{k}\left(y+(r / 2) v_{\omega-\pi / 2}\right) \\
& \geqslant u_{k}\left(y-(r / 2) v_{\omega-\pi / 2}\right)=u_{k}(x)
\end{aligned}
$$

This completes the proof.

Corollary 10.7 Let $\left\{u_{k}\right\}, \hat{u}, \delta, \epsilon=\epsilon_{\delta}, k_{\delta}$ be as in Theorem 10.5. If $k \geqslant k_{\delta}$, then the free boundary $\Gamma_{u_{k}}$ in $B_{1}^{\prime}$ is a Lipschitz graph.

Proof. This is a direct consequence of the directional monotonicity proven in Corollary 10.6 and the fact that $\Gamma_{u_{k}} \cap B_{1}^{\prime} \subset S_{\epsilon}$. 
COROLLARY 10.8 Let $\left\{u_{k}\right\}, \hat{u}, \delta, \epsilon=\epsilon_{\delta}, k_{\delta}$ be as in Theorem 10.5. If $k \geqslant k_{\delta}$ and $y \in \Gamma_{u_{k}} \cap B_{1}^{\prime}$, then $y$ is a regular point.

Proof. By Corollary 10.8 we know the free boundary can be represented by a graph. Recalling that we are in dimension three, by Theorem 9.7 we conclude that every free boundary point is regular.

Remark 10.9. The previous corollary shows that for a minimizer $u$, the set of regular points is relatively open in the free boundary $\Gamma_{u}$. This is seen by letting $u_{r_{k}} \rightarrow u_{0}$ be a blow up of $u$ at a regular point $x_{0}$ for some sequence $r_{k} \rightarrow 0$. Then $u_{0}$ is a rotation of the half-plane solution $\hat{u}$ and we may apply Corollary 10.8 to obtain that $\Gamma_{u_{r}} \cap B_{1}^{\prime}$, for $r=r_{k_{\delta}}$, consists only of regular points. Rescaling backwards, we obtain that all points in $\Gamma_{u} \cap B_{r}^{\prime}\left(x_{0}\right)$ are regular.

We also note that the set of regular points is relatively dense in $\Gamma$. This is seen by noting that free boundary points that have tangent balls from one side (as defined in [8]) are dense in $\Gamma$. It has been shown in [8] that free boundary points that have a tangent ball are regular.

COROLlaRY 10.10 (Differentiability) If $x_{0}$ is a regular point for a minimizer $u$, then the blow-up of $u$ at $x_{0}$ is unique. Moreover, if $u_{0}$ is the blow-up of $u$ at $x_{0}$, then $\Gamma_{u}$ has a tangent line at $x_{0}$ which is parallel to $\Gamma_{u_{0}}$.

Proof. Let $u_{0}$ and $v_{0}$ be two different blow-ups of $u$ at $x_{0}$. By Corollary 9.6, we know that $u_{0}$ and $v_{0}$ are both rotations of the half-plane solution $\hat{u}$. Without loss of generality assume that $u_{0}=\hat{u}$ and that $v_{0}$ is a rotation of $\hat{u}$ by angle $\alpha \in(0,2 \pi)$ in $\left(x_{1}, x_{2}\right)$-plane. By Corollary 10.6, $u$ will inherit directional monotonicity from both $\hat{u}$ and $v_{0}$. Specifically, in a small ball around $x_{0}, u$ will be monotone nondecreasing in directions $e_{\omega}=(\cos \omega, \sin \omega, 0)$ for

$$
\omega \in(\delta-\alpha, \pi / 2-\delta-\alpha) \cup(\pi / 2+\delta-\alpha, \pi-\delta-\alpha) .
$$

Then $\hat{u}$, being a blow-up of $u$, will also be monotone nondecreasing in those directions. However, if $\delta>0$ is small enough, the above range contains an angle $\omega \in(\pi, 3 \pi / 2) \cup(3 \pi / 2,2 \pi)$ for which $\hat{u}$ is strictly decreasing (where positive) in the corresponding direction $e_{\omega}$. This is clearly a contradiction, which implies the uniqueness of the blow-up.

To show the differentiability of $\Gamma_{u}$ at $x_{0}$, without loss of generality assume $x_{0}=0$. Then observe that the uniqueness of the blow-up implies that $u_{r} \rightarrow \hat{u}$ locally in $\mathbb{R}^{3}$ as $r \rightarrow 0$, not just only over a sequence. In particular, using the $C^{\alpha}$ convergence and the thin nondegeneracy as in the proof of Theorem 5.9, we will have that for any $\eta>0$, there exists $r_{\eta}>0$ such that $\Gamma_{u_{r}} \cap B_{1}^{\prime} \subset S_{\eta}$, for $r<r_{\eta}$. Rescaling backwards, we will have that

$$
\Gamma_{u} \cap B_{r}^{\prime} \subset S_{r \eta} \cap B_{r}^{\prime}, \quad r<r_{\eta} .
$$

This implies that $\Gamma_{u}$ (which is a Lipschitz curve) is differentiable at the origin and that $\Gamma_{\hat{u}}=\left\{x_{2}=\right.$ $\left.x_{3}=0\right\}$ is its tangent line.

We are now ready to prove Theorem 10.1. We only state what remains to be proven.

THEOREM 10.11 ( $C^{1}$ regularity) Let $u$ be a minimizer in dimension $n=3$ and $0 \in \Gamma$ a regular point. Then there exists a small $\rho>0$ such that $\Gamma \cap B_{\rho}^{\prime}$ is a $C^{1}$ graph.

Proof. Without loss of generality we may assume that the blow-up at the origin is the half-plane solution $\hat{u}$. Let $\delta>0$ and $\epsilon=\epsilon_{\delta}$ be as in Theorem 10.5. Then by using Corollary 10.7 and 
rescaling backwards, we obtain that there is a small $\rho>0$ such that $\Gamma_{u} \cap B_{\rho}^{\prime}$ is a Lipschitz graph. Furthermore, $\Gamma_{u} \cap B_{\rho}^{\prime} \subset S_{\rho \epsilon} \cap B_{\rho}^{\prime}$, and by Corollary 10.6, $u$ is monotone nondecreasing in $S_{\rho \epsilon} \cap B_{\rho}^{\prime}$ in directions $e_{\omega}$ for $\omega \in(\delta, \pi / 2-\delta) \cup(\pi / 2+\delta, \pi-\delta)$. By Corollary 10.8, we know that every point $y \in \Gamma_{u} \cap B_{\rho}^{\prime}$ is regular and, by Corollary 10.10, the blow-up $v_{0}$ of $u$ at $y$ is unique. Also, $v_{0}$ will inherit monotonicity in the direction $e_{\omega}$ for $\omega \in(\delta, \pi / 2-\delta) \cup(\pi / 2+\delta, \pi-\delta)$. Since $v_{0}$ must be a rotation of $\hat{u}$, the directional monotonicity implies that the rotation angle $\alpha$ must satisfy $|\alpha| \leqslant \delta$. In particular, the tangent line at $y$, parallel to $\Gamma_{v_{0}}$ will form an angle $|\alpha| \leqslant \delta$ with $\Gamma_{\hat{u}}$, which is the tangent line at the origin. This proves the $C^{1}$ regularity of $\Gamma_{u}$ at the origin. Repeating the last argument at every point of $\Gamma_{u} \cap B_{\rho}^{\prime}$, we obtain the $C^{1}$ regularity of $\Gamma_{u} \cap B_{\rho}^{\prime}$.

Acknowledgements. The authors are supported in part by NSF grant DMS-1101139

\section{REFERENCES}

1. Alt, H. W. \& CAfFarelli, L. A., Existence and regularity for a minimum problem with free boundary, J. Reine Angew. Math. 325 (1981), 105-144. Zb10449. 35105 MR0618549

2. Alt, H. W., Caffarelli, L. A. \& Friedman, A., Variational problems with two phases and their free boundaries, Trans. Amer. Math. Soc. 282 (1984), 431-461. Zb10844. 35137 MR0732100

3. Bouchaud, J.-P. \& Georges, A., Anomalous diffusion in disordered media: statistical mechanisms, models and physical applications, Phys. Rep. 195 (1990), 127-293. Zbl0816. 76089 MR1081295

4. Brascamp, H. J. , Lieb, E. H. \& LutTinger, J. M., A general rearrangement inequality for multiple integrals, J. Functional Analysis 17 (1974), 227-237. Zb10286. 26005 MR0346109

5. Caffarelli, L. A., Lederman, C., \& Wolanski, N., Uniform estimates and limits for a two phase parabolic singular perturbation problem, Indiana Univ. Math. J. 46 (1997), 453-489. Zb10909. 35012 MR1481599

6. Caffarelli, L. A. \& Salsa, S., A geometric approach to free boundary problems, Graduate Studies in Mathematics, vol. 68, American Mathematical Society, Providence, RI, 2005. Zbl1083. 35001 MR2145284

7. Caffarelli, L. A. \& Silvestre, L., An extension problem related to the fractional Laplacian, Comm. Partial Differential Equations 32 (2007), 1245-1260. Zb11143. 26002 MR2354493

8. Caffarelli, L. A. \& Roquejoffre, J. M., \& Sire, Y., Variational problems for free boundaries for the fractional Laplacian, J. Eur. Math. Soc. (JEMS) 12 (2010), 1151-1179. Zb11221. 35453 MR2677613

9. Jerison, D. S. \& KeniG, C. E., Boundary behavior of harmonic functions in nontangentially accessible domains, Adv. in Math. 46 (1982), 80-147. Zbl0514.31003 MR0676988

10. KAWOHL, B., Rearrangements and convexity of level sets in PDE, Lecture Notes in Mathematics, vol. 1150, Springer-Verlag, Berlin, 1985. Zb10593.35002 MR0810619

11. Malý, J. \& Ziemer, W. P., Fine regularity of solutions of elliptic partial differential equations, Mathematical Surveys and Monographs, vol. 51, American Mathematical Society, Providence, RI, 1997. Zb10882.35001 MR1461542

12. WeISS, G. S., Partial regularity for weak solutions of an elliptic free boundary problem, Comm. Partial Differential Equations 23 (1998), 439-455. Zb10897. 35017 MR1620644

13. Zaslavsky, G. M., Chaos, fractional kinetics, and anomalous transport, Phys. Rep. 371 (2002), 461-580. Zb10999.82053 MR1937584 